\title{
HIV und die Konsequenzen für Ernährungssicherheit und Gesundheit von Kindern in den betroffenen Gemeinden
}

\author{
Noel W. Solomons ${ }^{\mathrm{a}} \quad$ Gwen Ellen O'Donnell ${ }^{\mathrm{b}}$ \\ ${ }^{a}$ Center for Studies of Sensory Impairment, Aging and Metabolism, Guatemala Stadt, Guatemala, und \\ ${ }^{b}$ Project Concern International, Washington, D.C., USA
}

\section{Schlüsselwörter}

Humanes Immunschwäche-Virus (HIV) • Erworbenes Immunschwächesyndrom • Ernährungsunsicherheit • Ernährungssicherheit • Entwicklungsländer • Landwirtschaft • Nahrungsmittelhilfe - Opportunistische Infektionen • Armut

\footnotetext{
Zusammenfassung

Die ersten Fälle des durch das humane ImmunschwächeVirus (HIV) verursachten, erworbenen Immunschwächesyndroms (AIDS) wurden 1981 diagnostiziert. Inzwischen sind weltweit schätzungsweise 39 Millionen Menschen mit dem Virus infiziert. Während derzeit $64 \%$ dieser Menschen in den armen Regionen Subsahara-Afrikas leben, wird die schnellste Ausbreitung der Pandemie inzwischen in den grössten Ländern Asiens verzeichnet. Ernährungssicherheit besteht dann, wenn alle Menschen jederzeit physisch und wirtschaftlich Zugang zu einer ausreichenden Menge gesundheitlich unbedenklicher und nahrhafter Nahrung haben, um ihre Ernährungsbedürfnisse und Nahrungsmittelpräferenzen für ein aktives und gesundes Leben befriedigen zu können. Im Rahmen der HIV/AIDS-Pandemie ergaben sich aufgrund der hinlänglich bekannten, gesetzmässigen Wechselwirkung zwischen Mangelernährung und Infektion Bedenken hinsichtlich der Ernährungssicherheit. Schlechter ernährte Menschen leiden stärker unter Krankheitserregern und die verheerenden Auswirkungen der Infektionskrankheiten haben in der Tendenz einen Abbau der Nährstoffreserven zur Folge. Die sozialen und klinischen Folgen von HIV/AIDS gefährden die Nahrungsversorgung in den bereits von Armut und Umweltschädigung betroffenen Regionen. Interventionen, die auf landwirtschaftliche Produktivität, Einkommensgenerierung und Nahrungsmittelhilfe abzielen, müssen sowohl entwickelt als auch an die lokalen, gemeindespezifischen Gegebenheiten angepasst werden, und man muss sich vor Augen führen, dass diese Interventionen notwendig sind, um die nega-
}

tiven Auswirkungen von HIV/AIDS auf die Ernährungssicherheit abzuschwächen. Die UNICEF hat die Betreuung und Versorgung zu einem Teil ihrer Bemühungen gemacht, das Überleben von Kindern zu sichern, wobei Betreuung und Versorgung wie folgt definiert werden: Die auf Haushalts- und Gemeindeebene aufgewendete Zeit, Aufmerksamkeit und Unterstützung, um die körperlichen, geistigen und sozialen Bedürfnisse des heranwachsenden Kindes und anderer Haushaltsmitglieder zu erfüllen. Die Betreuung und Versorgung umfasst die medizinische Versorgung von Frauen, Stillen und Fütterungsmethoden von Kindern, psychosoziale Betreuung, Nahrungszubereitung sowie Hygiene- und Gesundheitspraktiken im Haushalt. Bei HIV-positiven Kindern bedient man sich inzwischen der positiven Abweichung, d.h. man identifiziert auf Haushaltsebene die Betreuungs- und Versorgungsmethoden, mit denen die besten Ergebnisse bezüglich des Wachstums, der Gesundheit und der Entwicklung der Kinder erzielt werden. Die Stigmatisierung ist ein vorherrschender Faktor, der tendenziell zur Ausgrenzung von Haushalten mit mindestens einem HIV-positiven Mitglied führt und so den sozialen Zusammenhang erschwert, in dem für Ernährungssicherheit gesorgt und Betreuungsinterventionen implementiert werden können. Die Lücken in unserem Wissen bezüglich der Beziehung von Ernährungssicherheit und HIV/AIDS wurden inzwischen identifiziert. Noch zu erforschen ist u.a. wie wirksam die Nahrungsmittelhilfe tatsächlich die Folgen einer HIV-Infektion abschwächen kann und welche Wechselwirkungen zwischen der Ernährungssicherheit und der Wirksamkeit und Verträglichkeit der antiretroviralen Therapie bestehen. Forschungsarbeiten sind jedoch angesichts der knappen Finanzmittel ein gewisser Luxus, so dass die Erhebung und Kodifizierung nützlicher praktischer Erfahrungen, die auf den Erfahrungen beruhen, die derzeit von den vor Ort tätigen Mitarbeitern und Gemeinden gesammelt werden, für diese Interventionen von unschätzbarem Wert sind.

\section{KARGER}

Fax +4161306 1234

E-Mail karger@karger.ch

www.karger.com (c) 2008 Nestec Ltd., Vevey/S. Karger AG, Basel

0250-9652/07/0651-0009\$23.50/0

Online-Zugriff auf:

www.karger.com/and
Noel W. Solomons

CeSSIAM in Guatemala

PO Box 02-5339, Section 6163/Guatemala

Miami, FL 33102-5339 (USA)

Tel./Fax +502 2473 3942, E-Mail cessiam@guate.net.gt 


\section{Einleitung und geschichtlicher Zusammenhang der AIDS-Pandemie}

Am 5. Juni 1981 wurde erstmals bei homosexuellen Männern in Los Angeles über klinische Fälle einer zehrenden, zum Tode führenden Infektionserkrankung berichtet, die später mit der Bezeichnung erworbenes Immunschwäche-Syndrom oder AIDS belegt wurde [1]. Diese Krankheit wird durch das humane Immunschwäche-Virus (HIV) übertragen. Inzwischen vermutet man, dass das Virus 20 bis 40 Jahre vor diesem Zeitpunkt erstmals vom Affen auf den Menschen übertragen worden sein könnte und in Afrika ein eng begrenztes Reservoir gebildet hat [2]. Erst nachdem AIDS später auch in der Karibik, in Brasilien und in Subsahara-Afrika festgestellt wurde, war die internationale Ärzteschaft bezüglich einer Epidemie in Alarmbereitschaft versetzt [3].

Epidemien kamen in der Geschichte der Menschheit immer wieder vor. Die Pest (der schwarze Tod) wütete zwischen dem 11. und dem 15. Jahrhundert in mehreren Wellen und dezimierte die Bevölkerung in Europa um bis zu ein Drittel. In der Zeit vor John Snow, d.h. vor Mitte des 19. Jahrhunderts, wurde Europa ferner von tödlich verlaufenden Cholera-Epidemien heimgesucht [4]. Die Cholera ist bis heute in Teilen Südasiens holoendemisch [5]. Die Pocken waren von der Antike bis zu ihrer Ausrottung 1979 eine weltweite Pandemie [6]. Ferner traten immer wieder Influenzapandemien auf, wobei das schlimmste Beispiel wohl die zum Ende des 1. Weltkriegs, zwischen 1918 und 1919 aufgetretene Grippepandemie, die sogenannte Spanische Grippe, war; man schätzt, dass der Spanischen Grippe weltweit ungefähr 3 Millionen Menschen zum Opfer gefallen sind.

$\mathrm{Zu}$ den entscheidenden Faktoren der Krankheitsübertragung von Mensch zu Mensch zählt die Mobilität. AIDS ist in dieser Hinsicht wahrscheinlich die erste Epidemie im Zeitalter der Globalisierung. Vor Einführung des international Flugverkehrs war es sehr viel schwieriger, grössere Distanzen zurückzulegen, und die eingeschränkte Mobilität begrenzte die Ausbreitung ansteckender Krankheiten. Ferner drangen Tod und Leid, die durch Krankheit, Hungersnöte und Naturkatastrophen verursacht wurden, vor Einführung der elektronischen Kommunikation nicht in das Bewusstsein der Menschen, die ausserhalb der betroffenen Regionen lebten. Seit Anfang des 20. Jahrhunderts ist die Weltbevölkerung von 2 Milliarden auf inzwischen geschätzte mehr als 9 Milliarden gewachsen [7]; die Zahl der potenziellen Opfer übertragbarer Krankheiten ist also weltweit gestiegen. Dies alles sind greifbare Faktoren, die sich im Kontext einer modernen Pandemie, wie z.B. der AIDS-Erkrankung, abzeichnen.

Von den geschätzten 39 Millionen HIV-infizierten Menschen weltweit leben über 25 Millionen (64\%) in SubsaharaAfrika. Während sich HIV-infizierte Menschen in Industrienationen vor Ausbruch der AIDS-Erkrankung noch jahrelang guter Gesundheit erfreuen und ein produktives Leben führen können, ist dies bei einem HIV-Infizierten in Afrika weniger wahrscheinlich. Millionen Afrikaner erkranken und sterben frühzeitig und zwar nicht, weil es an fortschrittlichen Arzneimitteln fehlt, sondern aufgrund vermeidbarer, meist auf Mangelernährung zurückzuführender Gesundheitsprobleme.
HIV/AIDS befällt derzeit hauptsächlich Menschen in der produktiven Altersgruppe, dezimiert somit die zur Verfügung stehenden Arbeitskräfte und wirkt sich dadurch wiederum stark auf das Auskommen und die Nahrungsmittel- und Ernährungssicherheit aus [8].

HIV/AIDS hat ausserdem erschreckende Folgen für Kinder und die hinsichtlich des Überlebens von Kindern erzielten Fortschritte. Laut Walker und Schwartländer [9] stieg die Zahl der durch HIV-Infektionen verursachten Todesfälle in der Altersgruppe der unter 5-Jährigen in Subsahara-Afrika von 2 auf $7,7 \%$. In fünf Ländern sterben über 30 von 1000 und in 16 Ländern zwischen 10 und 25 von 1000 der unter 5-Jährigen aufgrund einer HIV-Infektion. In den restlichen, insgesamt 18 afrikanischen Ländern liegen die Raten bei unter 10 von 1000 [9]. HIV/AIDS wirkt sich in Afrika zunehmend auf die Sterblichkeit der unter 5-Jährigen aus und ist zwar nicht der einzige, aber doch einer der Faktoren, welche die im Kampf um das Überleben von Kindern erzielten Fortschritte untergraben. Laut einer Studie von USAID macht HIV/AIDS jahrelange wirtschaftliche und soziale Entwicklungen zunichte [10]. In der gleichen Studie wird ferner die Tatsache ins Feld geführt, dass die Mortalitätsrate von Eltern, die Waisen hinterlassen, die noch im Jahr 1990 bei 16,4\% lag, bis zum Jahr 2010 auf mindestens $68,4 \%$ ansteigen wird. Diese Zahlen sind zwar erschütternd, beinhalten jedoch noch nicht jene Kinder, die bei kranken Eltern leben und die Rolle der Hauptversorger für Eltern, jüngere Geschwister und andere übernehmen müssen, bzw. Kinder, die die Verantwortung für das Familienauskommen tragen. Kinder in Afrika sind heutzutage mit Herausforderungen von unvergleichbarem Umfang und unvergleichbarer Komplexität konfrontiert.

Am 26. Juni 1945 wurden die Vereinten Nationen in San Francisco, USA, ins Leben gerufen. Mit der Gründung der Vereinten Nationen galten Armut, Elend und Krankheit nicht länger als unvermeidliche Folgen des menschlichen Daseins, und das Ungleichgewicht bezüglich der Wohlstandsverteilung wurde nicht länger als Teil der natürlichen sozialen Ordnung toleriert. Im Dezember 1948 wurde die Allgemeine Erklärung der Menschenrechte auf der dritten Vollversammlung der Vereinten Nationen verabschiedet. Ihre Bedeutung für Menschen, die mit HIV/AIDS leben, wurde Gegenstand vieler Kommentare [11]. Noch aktueller sind die von der UN formulierten Millennium-Ziele zur Förderung der Gesundheit und Entwicklung, von denen mehrere unmittelbar für HIV/AIDS relevant sind [12].

Dies sind nur einige der mit dem Vierteljahrhundert der AIDS-Pandemie assoziierten Meilensteine und historischen Zusammenhänge. Die meisten Ärzte und Gesundheitsbehörden betrachten Gesundheitsprobleme jedoch nicht durch das Prisma der Makropolitik, sondern eher auf Gemeindeebene oder über ihren täglichen Umgang mit Patienten. Wir sind daher davon ausgegangen, dass die Leser der Annales Nestlé sich insbesondere für die Gemeindeebene interessieren. «Nahrung, Betreuung und Gesundheit gelten weithin als die Dreifaltigkeit der Ernährung», kommentierte Haddad [13] anlässlich des 17. International Congress on Nutrition im Jahr 2001. In unserem Artikel zum Thema «HIV und die Konsequenzen für Ernährungssicherheit und Gesundheit von Kindern in den betrof- 
fenen Gemeinden» versuchen wir, angesichts der vielschichtigen biologischen, sozialen und psychologischen Auswirkungen der HIV-Endemizität, Einblicke zu geben in die in armen Gemeinden herrschenden Wechselwirkungen von Nahrungsaufnahme einerseits und Betreuung und Versorgung andererseits.

\section{Die Wechselwirkungen zwischen Ernährung und Infektion}

Die wichtigsten unmittelbaren Ursachen für Mangelernährung sind unzureichende Nahrungsaufnahme und Infektionserkrankungen [8]. Da es sich bei HIV/AIDS um einen Komplex bestehend aus einer primären Virusinfektion (HIV) in Verbindung mit opportunistischen Infektionen (AIDS) handelt, besteht eine wesentliche Wechselwirkung zwischen Mangelernährung und Infektion. Der jüngste Bericht des USInnenministeriums zu Nahrung und Ernährung von Menschen, die mit HIV/AIDS leben [14], konstatiert: «In vielen Teilen der Welt, insbesondere in Subsahara-Afrika, liegt eine hohe Prävalenz von HIV/AIDS und Mangelernährung vor. Die Auswirkungen hängen miteinander zusammen und verstärken sich in einer Art Teufelskreis gegenseitig.»

\section{Konzepte und Grundsätze}

Die konzeptionellen und wissenschaftlichen Grundlagen der biologischen Bedenken bezüglich der Ernährungsunsicherheit in Kombination mit einer Infektionserkrankung, wie z.B. HIV, reichen fast ein halbes Jahrhundert zurück. Scrimshaw et al. [15] dokumentierten unzählige, in der Natur auftretende Interaktionen zwischen Wirt und Pathogen, bei denen der Ernährungsstatus des Wirts die Reaktion auf eine Infektion bedingt und sich das Vorliegen einer Infektion auf den Ernährungsstatus des Wirts auswirkt. Diese Daten wurden 1968 in einer von der WHO veröffentlichten Reihe von Fachmonographien ergänzt [16]. Ein schlecht ernährter Wirt ist bei Exposition gegenüber einem Krankheitserreger anfälliger für die Infektion bzw. die Infektion dauert bei ihm länger, ist schwerer und verläuft eher tödlich. Wir wissen inzwischen, dass die allgemeine Grundlage für diese Wirkung des Ernährungsstatus mit einem intakten und funktionierenden Immunabwehrsystem des Wirts zusammenhängt. Ein entsprechender Nährstoffstatus könnte sich auf die erfolgreiche Immunabwehr des Wirts gegen den Krankheitserreger auswirken $[17,18]$. Dies trifft auf jeder Ebene zu, von der Undurchlässigkeit der Barrieren (Haut, Schleimhäute, Darmschleimhaut), die das Eindringen von Krankheitserregern verhindern, über die angeborenen Reaktionen, bei denen Krankheitserreger umschlossen und lysiert werden bzw. zerfallen, bis zu den erworbenen Immunantworten, wie z.B. antikörpervermittelte und systemisch-entzündliche Reaktionen.

Andererseits können Tiere oder Menschen, die an akuten oder chronischen Infektionen leiden, an Gewicht verlieren, ihr allgemeiner Ernährungsstatus kann sich verschlechtern und es kann zu einem spezifischen Vitamin- oder Mineralstoffmangel kommen. Die Lungentuberkulose wurde nicht umsonst früher als «Schwindsucht» bezeichnet. Die verheerenden Auswirkungen einer Infektion hemmen den Appetit und die Nährstoffabsorption bzw. zerstören oder vergeuden die Nährstoffe im Körper und führen so beim Wirt zu einer Verschlechterung des Ernährungsstatus. Eine akute Dehydratation bei Gastroenteritis beispielsweise ist die Folge einer Kombination aller der oben genannten Mechanismen. Seit langem weiss man auch, dass der schlimmsten Form der Proteinmangelerscheinung beim Menschen, die unter der Bezeichnung Kwashiorkor bekannt ist, häufig eine Masernerkrankung vorangeht. Diese beiden Wechselwirkungen zwischen Ernährung und Infektion gelten als synergistisch.

Umgekehrt zeigt ein gut genährter Wirt in manchen Fällen eine höhere Widerstandskraft gegen Infektionen bzw. Infektionen verlaufen bei ihm in abgeschwächter Form. Es gibt ausserdem Fälle, in denen ein überernährter Wirt anfälliger für unerwünschte Infektionen ist als ein normalernährter Wirt. Hier handelt es sich um Beispiele für sogenannte antagonistische Wechselwirkungen zwischen Ernährung und Infektion. In den 50er und 60er Jahren des 20. Jahrhunderts galten Adipositas und Überernährung weder in den Industrienationen noch in den Entwicklungsländern als wichtige Probleme des allgemeinen Gesundheitswesens. Zwar waren die mit Abstand meisten Interaktionen synergistischer Natur, aber zwischen Ernährung und Infektion gab es auch einige wenige antagonistische Wechselwirkungen, bei denen der Mangel eines Nährstoffs den Wirt vor einer Infektion bzw. vor den Folgen einer durch einen Krankheitserreger hervorgerufenen Infektion schützte $[15,16]$. Diese antagonistischen Wechselwirkungen traten im Allgemeinem bei intrazellulären Pathogenen auf, d.h. jenen Krankheitserregern, die in die Zellen des Wirts eindringen und sich mit den im Zellplasma vorhandenen Nährstoffen ernähren. In diesen Fällen gilt, je besser die Nährstoffreserven in den Wirtszellen desto attraktiver ist der Wirt für den Krankheitserreger und desto besser gedeiht dieser.

\section{Die synergistische Wirkung von HIV/AIDS auf den Ernährungsstatus}

Selbst bei flüchtigster Berücksichtigung dessen, was wir über die Progression der symptomatischen AIDS-Erkrankung wissen, ergibt sich ein klassisches Beispiel für die negativen Auswirkungen einer Infektion auf den Ernährungsstatus, wobei die HIV-Infektion die primäre, zugrundeliegende Erkrankung ist. Die Kachexie ist kennzeichnend für AIDS [19] und wurde in den afrikanischen Gebieten, in denen die Erkrankung zuerst beschrieben wurde, in der Sprache der Einheimischen auch als Abmagerungskrankheit (slim disease) bezeichnet. Auch die mit dem Vollbild AIDS verbundenen Infektionen, u.a. konventionelle Infektionen, wie z.B. Tuberkulose, aber auch sogenannte «opportunistische» Infektionen (d.h. Organismen, die bei einem normal ernährten Wirt in der Regel nicht pathogen sind) sind pathognomonisch für die AIDS-Erkrankung. Die Menschen, die mit AIDS leben, leiden aufgrund der HIV-Infektion und den damit verbundenen Infektionen 
unter Appetitmangel und einer milden bis stark ausgeprägten Anorexie. So kann beispielsweise der allgegenwärtige Dünndarmparasit Cryptosporidium parvum, der in der Regel bei immunkompetenten Personen nicht pathogen ist (bzw. bei Kleinkindern schlimmstenfalls eine selbstlimitierende Durchfallerkrankung hervorruft), zu einer anhaltenden, praktisch nicht behandelbaren Darminfektion führen, die mit Durchfallneigung und bis zu zwei Litern Stuhlverlust täglich einhergehen kann [20]. Die AIDS-Erkrankung wird mit einer chronischen Aktivierung der akuten Immunreaktion in Verbindung gebracht [21], die durch Fieber und ein Stoffwechselmuster gekennzeichnet ist, bei dem Makro- und Mikronährstoffe konsumiert werden und es zur Auszehrung der inneren Organe sowie zum Muskelabbau kommt. Da eine HIV-Infektion in allen Stadien mit Nährstoffverlust einhergeht, ist es von äusserster Wichtigkeit, dass infizierten Personen mit einer ausreichenden Nährstoffmenge versorgt und alle Massnahmen ergriffen werden, damit der Körper diese Nährstoffe behält.

\section{Die synergistische Wirkung des Ernährungsstatus auf die Infektion im Falle von HIV/AIDS}

Ausgehend von den Grundsätzen der Wechselwirkungen zwischen Ernährung und Infektion hoffte man zu Beginn des Zeitalters von HIV/AIDS, dass eine Verbesserung des Ernährungsstatus der armen und unterernährten Populationen zur Prävention der Virusübertragung beitragen könnte und zwar entweder der Virusübertragung zwischen den Sexualpartnern beim Geschlechtsverkehr [22] oder der vertikalen Transmission von der Mutter auf den Säugling [23-25]. Trotz der ursprünglichen Beobachtungen, dass die AIDS-Pandemie anfangs hauptsächlich wohlgenährte Homosexuelle aus reichen Industrienationen betraf, hoffte man, die Übertragung des Virus durch den Geschlechtsverkehr eindämmen zu können und stellte diesbezügliche Untersuchungen an. Die Supplementierung von Vitaminen oder Mineralstoffen (Mikronährstoffen) zur Prävention der mittels Geschlechtsverkehr übertragenen Neuinfektionen erzielte im Allgemeinen keine positiven Ergebnisse. Ähnliche Bestrebungen, den Mikronährstoffstatus der Mutter zu verbessern, um die Übertragung des Virus von der Mutter auf den Feten, das Neugeborene oder das Kleinkind zu senken, wurden durch die in der Praxis vor Ort tätigen Forscher in Erwägung gezogen. Der Artikel von Downs und Cooper [26] setzt sich kritisch mit den in diesen Untersuchungen gewonnenen Erkenntnissen auseinander.

Ferner riefen bestimmte Zusammenhänge zwischen mit der Nahrung aufgenommenen Mikronährstoffen und der Progression der HIV-Infektion zum Vollbild AIDS bzw. zum Tod [2730] Erwartungen hervor, dass die Infektion durch Reduzierung des Nährstoffmangels gelindert und ihre Progression bzw. der tödliche Verlauf der Erkrankung vermindert werden könnte. Am meisten Interesse wurde in diesem Zusammenhang dem Nährstoff Vitamin A entgegengebracht. Der Umfang des experimentellen Interventionsdesigns und die in den Feldstudien gewonnenen Erkenntnisse bezüglich des Mikronährstoffstatus, der Mikronährstoffsupplementierung und der Outcomes von HIV/AIDS werden umfassend von Fawzi behandelt [31].
Eine Steigerung der Virulenz des Retrovirus stellt eine theoretische, bislang jedoch unerforschte Möglichkeit in der komplexen Beziehung zwischen HIV und dem Ernährungsstatus des Wirts dar. Ein Modell für ein solches Szenario findet sich in der Arbeit von Beck et al. [32-35] in den USA. Diese Forschergruppe fand heraus, dass ein Coxsackie-Virus, das bei wohlgenährten Mäusen eine leichte, vorübergehende Atemwegsinfektion hervorruft, bei einem unter Selenmangel leidenden Mäusewirt zu einem virulenten, tödlichen Stamm mutiert [32]. Das mutierte Virus wird dann ferner virulent und verursacht auch bei Nagetieren mit adäquatem Selenstatus eine tödliche Infektionskrankheit. Man vermutet, dass auch andere Virenstämme bei Passage durch Wirte mit Nährstoffmangel mutieren und die Viren in der Folge eine gesteigerte Virulenz aufweisen könnten [34]. Angesichts der weltweit verbreiteten Mangelversorgung der Menschen mit Eisen, Jod, Vitamin A, Zink und anderen Nährstoffen muss das Mutationspotenzial des HI-Virus bei Wirten mit Nährstoffmangel unbedingt berücksichtigt und erforscht werden.

\section{Die antagonistische Wirkung des Ernährungsstatus auf die Infektion bei HIV/AIDS}

Wie alle Viren ist auch das Retrovirus, das AIDS verursacht, ein intrazelluläres Pathogen. Dies sollte eigentlich einige antagonistische Wechselwirkungen auf den Plan rufen, bei denen die Infektion durch Entzug eines für die Entwicklung und die Vermehrung des HI-Virus kritischen Nährstoffs verzögert werden könnte. Die mit dem Vollbild AIDS verbundenen Infektionen, u.a. die sogenannten «opportunistischen Infektionen», neigen ferner zur intrazellulären Vermehrung.

Dies führt zu einer Reihe wichtiger Überlegungen. Eine dieser Überlegungen betrifft die routinemässige Anreicherung oder Supplementierung eines Nährstoffs, der möglicherweise die Vermehrung des HI-Virus oder eines anderen eindringenden Pathogens begünstigt. Entfalten Programme, die auf eine Verbesserung des Mikronährstoffstatus abzielen und bei denen die Supplementierung der gesamten Bevölkerung verabreicht wird, in den Gemeinden, in denen HIV/AIDS endemisch auftritt, möglicherweise eine gemischte Wirkung? Wirken sich diese Programme auf manche Teile der Bevölkerung, beispielsweise HIV-negative Menschen mit einem Mangel an diesem Nährstoff, positiv, bei anderen Menschen, z.B. seropositiven Personen mit oder ohne Nährstoffmangel, jedoch negativ aus? Diese Frage gewinnt durch die Veröffentlichung der Ergebnisse einer routinemässigen Eisen- und Folsäuresupplementierung (mit oder ohne gleichzeitige Verabreichung von Zink) bei Kleinkindern aus einer Region der Insel Pemba, in Sansibar, Tanzania, in der die Malaria holoendemisch auftritt, zunehmend an Bedeutung [36]. Die in die beiden Behandlungsarme eingeteilten malariainfizierten Kinder, die eine Eisen- oder Folsäuresupplementierung erhielten, wurden tendenziell häufiger ins Krankenhaus eingewiesen und hatten eine höhere Mortalitätsrate als Kinder, die lediglich eine Zinksupplementierung erhielten oder in die Kontrollgruppen eingeteilt worden waren und nicht behandelt wurden (Placebo). 
Die Kehrseite des Themas bezieht sich auf das, was Weinberg [37] als «nutritional immunity», als «Nährstoff-Immunität», bezeichnet, d.h. die potenzielle Schutzwirkung, die ein leichter Nährstoffmangel in einer Umgebung entfalten kann, in der ein nährstoffabhängiges Pathogen übertragen wird. Könnte man die Vermehrung des HI-Virus mittels Nährstoffentzug hemmen, wenn man den Nährstoffbedarf des Virus kennen würde? Wäre ein HIV-infizierter Mensch widerstandsfähiger gegen opportunistische Infektionen, wenn sein Nährstoffstatus bezüglich der für die ursächlichen Bakterien bzw. Protozoen interessanten Nährstoffe eher an der untere Grenze liegen würde?

Die zuvor genannten Spurenelemente (Eisen und Zink) sind hinsichtlich der möglichen, mit einer Überdosierung verbundenen Gefahren von Interesse und werfen die Frage auf, ob eine allgemeine Intervention in Form einer Supplementierung dieser Nährstoffe für die gesamte Bevölkerung verträglich und klug ist. Bezüglich der Eisensupplementierung wurde eine Reihe theoretischer Gründe genannt, warum prall gefüllte Eisenspeicher im Körper intrazelluläre pathogene und opportunistische Organismen beherbergen [38]. Eine vor Kurzem in Kenia durchgeführte Interventionsstudie, bei der eine Eisensupplementierung erfolgte, ergab jedoch keine negativen Auswirkungen auf die HIV-Last [39].

Es wurde nachgewiesen, dass viele der viralen Mechanismen des HI-Virus zinkabhängig sind. Ferner beobachteten Tang et al. in ihren epidemiologischen Studien vor Einführung der antiretroviralen Therapie bei Männern, die über die Nahrung höhere Mengen an Zink zu sich nahmen bzw. eine Zinksupplementierung erhielten, eine stärkere Progression von HIV zu AIDS [27] und nach Ausbruch der AIDS-Erkrankung eine kürzere Überlebensdauer [28]. In einer jüngeren, in mancher Hinsicht ethisch etwas problematischen klinischen Studie in Durban, Südafrika, prüften Bobat et al. [38] die Hypothese, eine 6-monatige Verabreichung von $10 \mathrm{mg}$ elementarem Zink an jugendliche AIDS-Patienten in einem Kwa-Zulu-Natal Krankenhaus wirke sich negativ auf den Verlauf der Infektion aus. Bei den randomisiert in die mit Zink behandelte Gruppe eingeteilten Patienten wurde weder eine verstärkte Progression noch eine höhere Mortalität beobachtet; die Patienten sprachen im Gegenteil positiv auf die Zinksupplementierung an. Die andere Studie wurde in Kenia durchgeführt und von Olsen et al. [39] veröffentlicht. In dieser Studie erhielten seropositive schwangere Frauen zweimal wöchentlich eine Supplementierung mit $60 \mathrm{mg}$ Eisen und die Forschergruppe beobachtete ab Beginn der Supplementierung über einen Zeitraum von 4 Monaten die HIV-1-Last. Diese zweimal pro Woche verabreichte Eisensupplementierung wirkte sich nicht auf die Viruslast aus. Da 60-120 mg Eisen täglich in der Schwangerschaft jedoch eine normale Dosis darstellen, kann laut den Autoren eine «Wirkung höherer Dosen nicht ausgeschlossen werden» [39].

Die Ergebnisse der oben angeführten Studien bieten eine gewisse Sicherheit dafür, dass die Supplementierungs- oder Anreicherungsprogramme mit Zink oder Eisen auch für HIVpositive Kinder verträglich sind. Die theoretischen Überlegungen hinsichtlich des Wechselspiels von Eisen und Zink mit HIV oder anderen infektiösen Organismen beim AIDS-Syn-
Tabelle 1. Stellungnahme der American Dietetic Association (Amerikanische Gesellschaft für Ernährungswissenschaftler) and Dietitians of Canada zur Ernährungsintervention im Rahmen der Versorgung von Personen, die mit dem humanen Immunschwäche-Virus (HIV) infiziert sind

Die Infektion mit dem humanen Immunschwäche-Virus (HIV) und die Entwicklung des erworbenen Immunschwäche-Syndroms (AIDS) wirken sich signifikant auf die nationale und globale Gesundheit sowie die sozialen, politischen und wirtschaftlichen Outcomes aus. Präventions- und Behandlungsbemühungen, die auf eine Eindämmung der HIV-Infektion abzielen, sind schwieriger als in früheren Jahrzehnten. Die Gewährleistung von Nahrungsmittel- und Ernährungssicherheit und die Behandlung der mit der Ernährung verbundenen Komplikationen einer HIV-Infektion sowie die multiplen Aspekte der von einer HIV-Infektion ausgehenden oder mit dieser zusammenhängenden Erkrankung sind nach wie vor Herausforderungen für Patienten und alle, die an der Prävention, Pflege und Behandlung von HIV/AIDS mitwirken. Zu den irritierenden klinischen Problemen zählen u.a. Wechselwirkungen zwischen Medikamenten, gleichzeitig auftretende andere Infektionen und Erkrankungen, Abmagerung und Lipodystrophie.

Aus Fields-Gardner und Fergusson [44].

drom $[39,40]$ sprechen jedoch dafür, auch weiterhin wachsam zu bleiben und auf Anzeichen zu achten, die in Gegensatz zu diesen aus nur zwei Studien stammenden Ergebnissen stehen.

\section{Ernährungssicherheit und -unsicherheit in den von HIV/AIDS betroffenen Gemeinden}

Im Jahr 2001 bemerkte der damalige Leiter von UNAIDS, Dr. Peter Piot, dass die Versorgung mit einer ausreichenden Menge an Nahrungsmitteln die drängendste Sorge der von ihm besuchten mit HIV/AIDS lebenden Menschen sei [41]. Whiteside et al. [42] verweisen hinsichtlich des Zeitrahmens auf eine wichtige Tatsache, nämlich dass die lange Inkubationszeit zwischen Infektion und Ausbruch der Erkrankung eine Reihe von Konsequenzen hat. Im Klartext heisst dies: Es dauert lange bis sich HIV-Infektionen zu AIDS-Fällen entwickeln und aus AIDS-Fällen Todesfälle werden, die u.a. Verwaisung, Armut und veränderte Bevölkerungsstrukturen mit sich bringen. Inzwischen jedoch dauert die HIV/AIDS-Epidemie schon 25 Jahre, und wir können damit beginnen, diese Folgen im Kontext ihrer Auswirkungen auf die Ernährungssicherheit der betroffenen Populationen zu betrachten. Tabi und Vogel [43], zwei vor Ort in Ghana in der Pflege beschäftigte Forscher, stellen eine beunruhigende Behauptung auf: «In Ghana und in vielen anderen Entwicklungsländern sterben mehr HIV- und AIDS-Patienten aufgrund ihres schlechten Ernährungsstatus als an der Krankheit selbst.» Das 2004 verfasste Positionspapier der Gesellschaften der Ernährungswissenschaftler der USA und Kanada (Tabelle 1) stammt zwar aus den zwei reichsten Nationen des nordamerikanischen Kontinents, [44] vermittelt aber trotzdem die umfassende und globale Reichweite der Probleme, die sich im 21. Jahrhundert weltweit auf die Ernährungssicherheit und die Betreuung und Versorgung von Kindern auswirken. 


\section{Definitionen der Ernährungssicherheit und Messgrössen der Ernährungsunsicherheit}

Laut der Erklärung von Rom und dem Aktionsplan des Welternährungsgipfels der UN-Ernährungs- und Landwirtschaftsorganisation (FAO) besteht Ernährungssicherheit dann, «wenn alle Menschen jederzeit physisch und wirtschaftlich $\mathrm{Zu}$ gang $\mathrm{zu}$ ausreichender, gesundheitlich unbedenklicher und nahrhafter Nahrung haben, um ihre Ernährungsbedürfnisse und Nahrungsmittelpräferenzen für ein aktives und gesundes Leben befriedigen zu können» [45]. Ein Zustand der «Ernährungsunsicherheit» liegt also vor, wenn die oben angeführten Kriterien der Definition nicht erfüllt sind. Die Kurzform der Definition des Begriffs «Ernährungsunsicherheit» lautet «eingeschränkter oder unsicherer Zugang zu einer ausreichenden Menge nahrhafter Nahrung» [46]. Die Bedenken bezüglich der Ernährungssicherheit können sich auf verschiedene Ebenen beziehen, u.a. die nationale, die lokale (Gemeinde), die familiäre (Haushalt) und sogar die persönliche Ebene. Logischerweise beruht die Ernährungssicherheit auf der Menge an Nahrungsmitteln, die auf den diversen Ebenen verfügbar sind. Bei ungleicher Verteilung einer unzureichenden Menge an Nahrungsmitteln kann zwar bei manchen Menschen ein Zustand der Ernährungssicherheit erzielt werden, da aber der Begriff «Ernährungssicherheit» gerechterweise für «alle Menschen» gilt, müsste ein Land über eine Menge an Nahrungsmitteln verfügen, die ausreicht, um die Bedürfnisse aller seiner Einwohner decken zu können.

Diverse Institutionen und Personen haben versucht, die Definitionen in Formate zu operationalisieren, mittels derer sich die Ernährungssicherheit bewerten und ein Zustand der Ernährungsunsicherheit diagnostizieren lässt. Die UN-Ernährungsund Landwirtschaftsorganisation (FAO) sammelt und tabellarisiert für die meisten Länder Daten zur Ernährungsbilanz, mit deren Hilfe sie feststellen kann, ob in einem Land «Mangelernährung» vorliegt. Dies ist dann der Fall, wenn die Kalorienzahl der produzierten oder importierten Nahrungsenergie nicht ausreicht, um die Bedürfnisse der Bevölkerung zu decken. Die FAO bestand allgemein auf einer minimalistischen Definition, die auf der absolut notwendigen Mindestration basiert [45]. Andere Institutionen und Personen stellen detailliertere und ehrgeizigere Forderungen auf. So kommentiert Gopalan beispielsweise [47]: «Um auf Haushalts-ebene Ernährungssicherheit zu erzielen, muss auf nationaler Ebene eine ausreichende Nahrungsmenge zur Verfügung stehen und diese gerecht, gemäss der physiologischen Bedürfnisse in der Bevölkerung verteilt werden.»

Für die meisten der mit HIV/AIDS in Verbindung stehenden Praxis- und Forschungskategorien zum Thema Ernährungssicherheit eignet sich die Haushaltsebene (bzw. die persönliche Ebene) am besten. Die Instrumente, mit denen die Ernährungssicherheit in der Praxis bewertet wird, stammen noch aus den frühen 90er Jahren des 20. Jahrhunderts $[48,49]$ und umfassen Datenerhebungen zu demographischen Merkmalen, zur Häufigkeit des Verzehrs von Obst und Gemüse sowie zu der dem Haushalt zur Verfügung stehenden Menge an Nahrungsmitteln.
Eine Kreuzvalidierung der anderen Indikatoren für eine Unterversorgung mit Nahrungsmitteln in Haushalten in ländlichen Gebieten Amerikas ergab bezüglich der Feststellung der Ernährungsunsicherheit ein hohes Mass an Sensitivität und Spezifität. An anderer Stelle wird die Ernährungsdiversität als ein möglicher Indikator für Ernährungssicherheit und als ein Prädiktor für den Ernährungsstatus angeführt, wobei es hinsichtlich der Standardisierung der Definitionen und der Methodologie jedoch weiterer Forschungsbemühungen bedarf, bevor diese als allgemein gültig angewendet werden können [50]. Bisher wurden zusätzliche Instrumente, mit denen sich die Ernährungssicherheit auf Haushaltsebene messen lässt, weiter verfeinert; diese Instrumente haben sich in populationsbasierten Umfragen als zuverlässig erwiesen und einige Studien haben ergeben, dass zwischen dem Mass der Ernährungssicherheit und der Nährstoffaufnahme ein Zusammenhang besteht [51].

Obwohl die in der Praxis verwendeten Fragebögen in bestimmten Settings validiert und ihre Konstruktvalidität nachgewiesen wurde, ist die Bewertung der «Ernährungsunsicherheit» nicht frei von Paradoxa. Rose [52] untersuchte die Indikatoren der Ernährungssicherheit in einer national repräsentativen Stichprobe der US-Bevölkerung und fand heraus, dass die meisten Mitglieder von Haushalten, die als mit Nahrungsmitteln unterversorgt galten (interessanterweise jedoch nicht die Familienmitglieder im Kindergartenalter) geringere tägliche Nährstoffaufnahmen angaben als Mitglieder von Familien, in denen eine ausreichende Menge an Nahrungsmitteln zur Verfügung stand. Ferner nimmt die Prävalenz des Übergewichts im Kindesalter zu. Bei Mädchen im Alter zwischen 12-17 Jahren besteht laut der US Food Security Scale [46] ein Zusammenhang zwischen grösserer Ernährungsunsicherheit und Übergewicht. Shariff und Khor [53] wendeten das gleiche, im US-Bundesstaat New York validierte Instrument $[48,49]$ in ländlichen Regionen Malaysias an und kamen ebenfalls zu dem scheinbar paradoxen Ergebnis, dass die Prävalenz von Übergewicht und Adipositas bei Frauen aus Haushalten, in denen Ernährungsunsicherheit herrschte, höher war (50\%) als bei Frauen aus Haushalten mit guter Ernährungssicherheit (38\%). Der gleiche Zusammenhang wurde auch bezüglich des Hüftumfangs festgestellt.

Aus diesen Gründen wurde vor kurzem eine Bewegung ins Leben gerufen, die es sich zur Aufgabe gemacht hat, die Bewertungsziele, die Konzeptionalisierung und die Messung der Ernährungssicherheit zu überarbeiten [54]. Die Übersicht unterstreicht die drei wichtigsten konzeptionellen Entwicklungen im Rahmen der praktischen Ansätze zur Messung der Zwänge und Beschränkungen hinsichtlich der Nahrungsverfügbarkeit: (1) die Entwicklung, nicht länger die Verfügbarkeit und Verwendung der Nahrungsmittel, sondern eher den «unzureichenden Zugang zu den Nahrungsmitteln» zu messen; (2) die Verlagerung des Schwerpunkts von objektiven auf subjektive Messgrössen und (3) die zunehmende Betonung elementarer Messungen anstatt sich auf Ersatzgrössen («proxy measures») zu verlassen. Webb et al. [54] beschreiben eine Agenda mit vier Punkten (Tabelle 2) zur weiteren Verbesserung und Verfeinerung der Messung der Ernährungssicherheit. Angesichts der paradoxen Elemente, die den oben erläuterten, traditionellen 
Tabelle 2. Weitere Fragen, die es zur Bewertung der Ernährungssicherheit und -unsicherheit zu erforschen gilt

1 Wie gut erfassen die zur Bestimmung der Ernährungsunsicherheit auf Haushaltsebene ausgelegten Messgrössen, die für Umgebungen mit chronischer Ernährungsunsicherheit ausgelegt sind, die Vorgänge, die zur akuten Ernährungsunsicherheit führen und die diesbezüglich gemachten Erfahrungen?

2 Wie beeinflussen temporäre Katastrophen, wie z.B. starkes Hochwasser oder Erdbeben, die Verhaltensweisen auf Haushaltsebene, die für Reaktionen auf Fragen der Ernährungssicherheit massgeblich sind?

3 Wie lässt sich das Wechselspiel zwischen der Schwere der Konsequenzen und der Häufigkeit von Verhaltensweisen auf Haushaltsebene, die mit der Ernährungsunsicherheit zusammenhängen, besser messen?

4 Wie lässt sich feststellen, ob die Antworten eines Einzelnen auf die in einer Umfrage gestellten Fragen zum Thema Ernährungsunsicherheit repräsentativ für die Erfahrungen aller Haushaltsmitglieder sind?

Aus Webb et al. [54].

Tabelle 3. Die Auswirkungen des Verlusts an produktiven Arbeitskräften auf die landwirtschaftliche Produktivität

1 Abnahme der landwirtschaftlich genutzten Fläche, da die Menschen körperlich nicht in der Lage sind, auf dem Feld zu arbeiten

2 Verringerung der Ernteerträge, da sich bestimmte landwirtschaftliche Interventionen, wie z.B. ein Wechsel der Anbaustruktur, verzögern

3 Veränderung der Anbaustruktur, da einige Familien bereits auf weniger arbeitsintensive Anbaupflanzen umgestiegen sind

4 Geringere Vielfalt an Anbaupflanzen pro Haushalt, da die von AIDS betroffenen Familien sich auf den Anbau einer Hauptpflanze beschränken

5 Abnahme der Fähigkeit zur Schädlingsbekämpfung durch Jäten und andere Massnahmen zwischen den Anbauperioden aufgrund eines Mangels an Arbeitskräften

6 Verlust an landwirtschaftlichem Wissen und der zur Lenkung eines landwirtschaftlichen Betriebs benötigten Fertigkeiten aufgrund des aidsbedingten Todes eines oder beider Elternteile

7 Rückgang der Viehzucht, da einige Familien, möglicherweise aus dringender Geldnot, zum Verkauf von Tieren gezwungen sind

In abgeänderter Form übernommen von Saasa [60].

Ansätzen anhaften und der grösseren konzeptionellen Tiefe, scheint dieser neue Ansatz, ist er denn einmal entwickelt, hinsichtlich der Auswirkungen von HIV/AIDS vielversprechender und umfassender zu sein.

\section{Die Vulnerabilität armer Gemeinden}

Arme Gemeinden in Entwicklungsländern haben das höchste Risiko für eine erhöhte AIDS-Inzidenz und -Prävalenz $[55,56]$. Armut macht ferner anfällig für eine unzureichende Nahrungsaufnahme, einen gestörten Ernährungsstatus und eine beschränkte Gesundheitsversorgung $[57,58]$. Die zusätzlich zu den bereits schwierigen Umständen der Armut in den ländlichen und urbanen Gebieten auftretende HIVÜbertragung hat die Bewältigungsmechanismen, die vor Auftreten der AIDS-Pandemie vorhanden waren, vollständig ausser Kraft gesetzt.

HIV und AIDS betreffen alle Mitglieder einer Gesellschaft, nicht nur Personen, die mit dem Virus infiziert sind und mit dem Virus leben. Die Erkrankung weist ferner, wie von Whiteside et al. geschildert [42], einzigartige Merkmale auf: «Aufgrund der langen Inkubationszeit zwischen der Infektion und dem Ausbruch der Erkrankung dauert es lange, bis sich HIV-Infektionen zu AIDS-Fällen entwickeln und aus AIDSFällen Todesfälle werden, die u.a. Verwaisung, Armut und veränderte Bevölkerungsstrukturen mit sich bringen». Dies bedeutet ferner, dass es Jahre dauern wird, nachdem die HIVInfektion ihren Höchststand erreicht hat, bis sich die Auswirkungen der AIDS-Erkrankung vollumfänglich offenbaren - diese Epidemie ist langfristig. Eine generationsübergreifende, lebenslange Perspektive im Umgang mit den lokalen Manifestationen der Pandemie ist daher wichtig.» Zumindest in Afrika, dem Kontinent mit den meisten Menschen, die mit HIV/AIDS leben, ist auch die Ernährungsunsicherheit extrem problematisch und die Prognose hinsichtlich der anhaltenden und sich weiter ausdehnenden Armut äusserst erschreckend. Laut Rukuni [59] ist Afrika die einzige Region der Welt, in der die Zahl der Hunger leidenden Menschen auch im Jahr 2020 weiter ansteigen und sich auch die Zahl der unterernährten Kinder entsprechend erhöhen wird.

\section{Faktoren, welche die Ernährungssicherheit von Menschen bedingen, die mit HIV/AIDS leben}

Unabhängig von den möglichen Einschränkungen hinsichtlich der genauen Messung der Ernährungsunsicherheit enthält der nachfolgende Überblick die Bedingungen und Faktoren, die in bestimmten Umgebungen bzw. Gesellschaften den Zugang zu Nahrungsmitteln bzw. die Nahrungsmittelverfügbarkeit auf Gemeindeebene gefährden oder schützen. Inzwischen werden erste wissenschaftliche Studien zu der Frage durchgeführt, inwieweit sich diese Faktoren mit HIV-infizierten Personen bzw. Haushalten überschneiden.

\section{Verlust an Humankapital und landwirtschaftliche \\ Produktivität}

Die in einem Land verfügbare Nahrungsmenge ist ein Produkt der Landwirtschaft, des Gartenbaus oder des internationalen Handels. Für den Handel gilt, dass die Importfähigkeit von dem durch die Exporte geschaffenen Wohlstand abhängt, der wiederum eine Funktion der natürlichen und humanen Ressourcen ist, die zu werthaltigen Gütern und Dienstleistungen verarbeitet bzw. umgewandelt werden. Bei Landwirtschaft und Gartenbau erstreckt sich die Produktionsgrösse von Grossbetrieben bis zu Familienbetrieben. HIV/AIDS hat auf allen Ebenen der landwirtschaftlichen Aktivität eine Reihe negativer Auswirkungen. Saasa [60] hat diese Auswirkungen aus der Perspektive der in Afrika gemachten Erfahrungen aufgelistet (Tabelle 3). 
Am häufigsten wird die in Tabelle 3 an erster Stelle aufgeführte Auswirkung genannt, nämlich die Verringerung des Humankapitals, d.h. der Arbeitskräfte, und die damit einhergehende Abnahme der Landnutzung. Rutengwe kommentiert [8] wie folgt: «HIV/AIDS betrifft derzeit hauptsächlich Menschen in der jungen, produktiven Altersgruppe. Dies wirkt sich stark auf den Lebensunterhalt und die Ernährungs- und Nahrungsmittelsicherheit aus, da die Zahl der verfügbaren Arbeitskräfte abnimmt.» Die Zahl der verfügbaren Arbeitskräfte verringert sich zusätzlich, da die gesunden Menschen die Infizierten versorgen und den Begräbnissen der Verstorbenen beiwohnen müssen. Kinder müssen die Schule vorzeitig verlassen und haben daher ein niedrigeres Bildungsniveau. Mit dem vorzeitigen Ableben der erwachsenen Arbeiter geht ferner das von Generation zu Generation weitergereichte Wissen und das praktische Lernen unter der Aufsicht einer erfahreneren Person verloren. Laut der FAO der Vereinten Nationen hat AIDS/HIV bisher über sieben Millionen Menschen in 25 Ländern SubsaharaAfrikas das Leben gekosten und in den nächsten zwei Jahrzehnten werden weitere 16 Millionen Todesopfer erwartet.

Die Schwächung des Arbeitskräftepotenzials durch HIV/ AIDS kann sich negativ auf die Fähigkeit einer Nation auswirken, Nahrungsmittel zu produzieren bzw. zu importieren. Mehrere andere Auswirkungen lassen sich anhand von Beispielen der jüngeren Literatur illustrieren. Bereits 1993, nur 12 Jahre nach Ausbruch der HIV/AIDS-Epidemie, dokumentierte eine retrospektive, in den zwei fruchtbarsten Bezirken von Uganda durchgeführte Studie, dass die Landnutzung in $66 \%$ der befragten Haushalte zurückgegangen war und dass bei der Geflügel- und Rinderzucht ein entsprechender Rückgang von 11 bzw. $32 \%$ verzeichnet wurde, der auf schlechtes Management zurückgeführt wurde [60]. Tod und Krankheit wurden am häufigsten als Gründe für den Rückgang der Ernteerträge angeführt; Schätzungen gingen davon aus, dass in der Region, in der die Studie durchgeführt wurde, 8\% der Familien mit Kindern unter fünf Jahren von diesem Rückgang der Ernteerträge betroffen waren. Ferner hat sich vor diesem Hintergrund der Einsatz von Pestiziden, Herbiziden und Düngemittel aufgrund der Armut verringert.

Laut der Beschreibung von Gillespie et al. [62] hat das Auftreten von HIV/AIDS weitere Auswirkungen auf die Landnutzung, u.a. werden Pflanzen (z.B. einige Knollengewächse) angebaut, die weniger arbeitsintensiv aber auch weniger nahrhaft sind. Geht man davon aus, dass die Familie sich dies leisten kann, so wird Land, das brachliegt, für spätere Ernten zwar fruchtbarer, aber die Familien, insbesondere Frauen und Waisen, laufen grössere Gefahr, ihre Landrechte zu verlieren. Natürliches und physisches Kapital geht verloren, weil Familien gezwungen sind, produktive Vermögenswerte (d.h. Ausrüstung und Werkzeuge) und Viehbestand zu verkaufen, um die Arzt- und Beerdigungskosten zu bezahlen, was ihnen wiederum die Wiederaufnahme der landwirtschaftlichen Tätigkeit nahezu unmöglich macht. Ausserdem wirken sich auch andere Faktoren, u.a. die Überbevölkerung und die z.B. in Uganda beobachtete Aufteilung des Lands zwischen den Erben negativ auf die landwirtschaftliche Produktivität aus [61].

Ein weiterer mit der landwirtschaftlichen Produktivität verbundener Aspekt führt die Anforderungen des ländlichen, landwirtschaftlichen Lebens und dessen arbeits- und zeitintensive Aufgaben als einen der Faktoren an, der die äthiopischen Frauen daran hindert, an Aufklärungsmassnahmen bezüglich der Prävention von Geschlechtskrankheiten teilzunehmen [63].

\section{Die Probleme der Nahrungsmittelversorgung auf}

Haushaltsebene

Frauen sind sowohl in den ländlichen als auch in den städtischen Gebieten Afrikas, in denen AIDS endemisch ist, Teil des Arbeitskräftepotenzials. Eine an verheirateten Frauen in Malawi, Simbabwe und Sambia durchgeführte Studie untersuchte die Faktoren, die sich in den von AIDS/HIV betroffenen, dürrebedrohten Gebieten auf den Ernährungsstatus der Mütter auswirken [64], und erzielte je nach geographischer Region unterschiedliche Ergebnisse. Frauen in Malawi, die innerhalb der Familie wenig Entscheidungsbefugnisse hatten, und Frauen in den städtischen Gebieten Sambias hatten ein grösseres Risiko für chronische Unterernährung, die laut Definition ab einem Body-Mass-Index von $<18,5 \mathrm{~kg} / \mathrm{m}^{2}$ vorliegt. In Simbabwe ergab sich kein Zusammenhang zwischen der Körperzusammensetzung und der Entscheidungsbefugnis der Frauen. Die Autoren zogen den Schluss, dass Frauen mit wenig Befugnissen und chronischer Unterernährung möglicherweise an produktiver Kapazität einbüssen und dadurch vor dem Hintergrund einer hohen HIV/AIDS-Prävalenz einem grösseren Risiko für Ernährungsunsicherheit und HIV/AIDS ausgesetzt sind [64].

Die Ernährungssicherheit auf Haushaltsebene kann aufgrund unzureichender Finanzmittel, mangelnder Infrastruktur und einer falschen Aufteilung der Ressourcen zwischen den armen südafrikanischen Landarbeiterfamilien auch dann gefährdet sein, wenn die Eltern nicht krank sind [65]. Die daraus resultierende Ernährungsunsicherheit wirkt sich insbesondere auf Kinder negativ aus, von denen die Hälfte untergewichtig, im Wachstum zurückgeblieben oder abgemagert ist. Diese Daten stammen aus einer Studie, welche die verarmten Lebensbedingungen der Landarbeiterfamilien in den landwirtschaftlichen Gemeinden der nordwestlichen Provinz von Südafrika beleuchtet [65], können aber durchaus als typisch für die Situation im gesamten südlichen Afrika angesehen werden.

Die soziale Ausgrenzung und Stigmatisierung, die Diskriminierung und Meidung Einzelner und ihrer Familien sind Faktoren, die sich negativ auf die Fähigkeit eines Haushalts, Nahrung zu beschaffen, auswirken. Die Stigmatisierung aufgrund einer AIDS-Infektion ist von Gemeinde zu Gemeinde und von Gesellschaft zu Gesellschaft unterschiedlich [63, 6670], aber die Betroffenen werden im Allgemeinen gemieden. Selbst wenn eine infizierte Person körperlich in der Lage sein sollte, produktiv zu arbeiten, wird sie auf eine andere Art und Weise vom Kreis der Erwerbstätigen ausgeschlossen [67], und Nachbarn und Konsumenten meiden möglicherweise die hausgemachten Produkte von Infizierten. Die direkt von einer HIV-Infektion betroffenen Familien werden innerhalb einer bereits armen Gesellschaft zu den Ärmsten der Armen, und dies schränkt ihren Zugang zu den Finanzmitteln ein, die sie benötigen, um ihre Familien mit Nahrung und anderen Grundgütern zu versorgen [66]. 
Der Verlust an Humankapital wirkt sich, wie bereits erwähnt, verheerend auf die landwirtschaftliche Produktion aus. Das Gleiche gilt für ein Wirtschaftsumfeld am Existenzminimum. Die Bestellung einzelner Landparzellen, um Nahrung für den Haushalt zu produzieren, wird zunehmend schwieriger, je schlechter der Gesundheitszustand der infizierten Haushaltsmitglieder ist und je mehr sie an Kraft und Energie verlieren [71]. Den Arbeiten auf dem Feld kann keine Aufmerksamkeit mehr geschenkt werden und das Land liegt brach.

Neben dem Verlust an Human-, Natur- und physischem Kapital erleiden die Haushalte auch grössere Finanzverluste. Die mit der Stigmatisierung verbundenen Probleme hinsichtlich der Ablehnung offizieller Bankkredite und gruppenbasierter Mikrofinanzinitiativen sowie die Schwierigkeiten, Tagelöhner zu bekommen, verursachen weitere finanzielle Belastungen für die von HIV/AIDS betroffenen Haushalte, die sich in ihrer Verzweiflung gezwungen sehen, Kredite bei inoffiziellen Geldgebern aufzunehmen, die exorbitante Zinssätze verlangen.

Die Daten aus einigen Fallstudien illustrieren die finanziellen Belastungen, die den mit HIV/AIDS lebenden Menschen aufgebürdet werden. Laut einer Veröffentlichung des Jahres 2004 aus dem Tschad beliefen sich die Kosten, die den Haushalten eines mit AIDS-infizierten Menschen bis zu dessen Tod entstehen, auf 836 USD pro Fall [72]. 28\% dieser Gesamtkosten ergeben sich aus Produktivitätsverlusten. Mehr als die Hälfte der Gesamtkosten (56\%) wurden für die Gesundheitsversorgung und $16 \%$ für die Beerdigung aufgebracht. Haushalte mit AIDS-Patienten mussten ferner häufiger als die Kontrollhaushalte Wertgegenstände beleihen oder verkaufen, um die Behandlungskosten bezahlen zu können. Die Ausgaben der Haushalte mit AIDS-Patienten waren hauptsächlich aufgrund der Gesundheitskosten viel höher als die der Kontrollhaushalte.

\section{Probleme auf Haushaltsebene bezüglich der Ernährung von Säuglingen und Kleinkindern}

Beim Stillen von Säuglingen und Kleinkindern entstehen zwar keine offensichtlichen monetären Kosten, aber der Energiebedarf der stillenden Mütter steigt um ca. 500 kcal/Tag verglichen mit gleichaltrigen Frauen, die nicht schwanger sind und nicht stillen [73]. Obwohl abgesehen von den besonderen Anforderungen hinsichtlich der HIV-Infektion keine weiteren Anforderungen bezüglich des Stillens aufgestellt wurden [74], kann der Erwerb der Nahrung, die erforderlich ist, um den für das Stillen benötigten zusätzlichen Energiebedarf von $500 \mathrm{kcal}$ zu decken, in den Haushalten, in denen bereits die Deckung des Grundenergiebedarfs ein Problem darstellt, der Auslöser für die finanzielle Überforderung der Haushalte sein.

In Haushalten, in denen bereits der Erwerb zusätzlicher Nahrung für die Mutter ein Problem darstellt, ist spezielle Säuglingsnahrung noch unerschwinglicher. In den ärmsten Gemeinden gibt es möglicherweise gar keine Säuglingsmilchnahrung zu kaufen und falls es doch welche gibt, so ist sie für die Haushalte, die sich aufgrund einer HIV-Infektion der Mutter für diese Option entscheiden, möglicherweise unerschwinglich [75]. Entscheidet sich eine Mutter gegen das Stillen und für eine Muttermilchersatznahrung, dann belasten die Gesamtkosten für die Säuglingsmilchnahrung und die für das Füttern benötigten Utensilien die Haushaltsressourcen be- trächtlich. Angesichts der begrenzten Hygiene gilt die weitere Sorge der Verträglichkeit der Milchnahrung, die in unsicheren hygienischen Verhältnissen hergestellt wird [76], und es wird ein Anstieg der Durchfallepisoden bei Kindern prognostiziert. Dies verursacht weitere Aufwände im Bereich der Betreuung und Versorgung von Kindern, Kosten für Medikamente sowie ein erhöhtes Risiko der Säuglingssterblichkeit, die zu den «Kosten» des Nichtstillens addiert werden müssen. Zu guter Letzt belastet jede für die Säuglingsmilchnahrung getätigte Ausgabe die für die Ernährung der älteren Geschwister zur Verfügung stehenden Mittel.

Das nächste Stadium in der Entwicklung der kindlichen Ernährung, in der sich die Ernährungssicherheit auswirken kann, ist die Einführung der «Beikost». Die Ernährung, die den Säuglingen in armen Haushalten als Ergänzung oder Ersatz für die Muttermilch geboten wird, ist einseitig und schwer verdaulich und deckt nur selten den Energie- und Nährstoffbedarf, der für ein schnelles Wachstum, zum Auffüllen der Nährstoffspeicher und zum Aufbau der Widerstandskraft gegen Infektionserkrankungen benötigt wird und bisher von der Muttermilch gedeckt wurde [50]. Das in Subsahara Afrika beobachtete, typische Wachstum während der ersten fünf Lebensjahre und die ab der Geburt beobachtete Prävalenz der Unterernährung lassen darauf schliessen, dass die Kinder über die Nahrung unzureichend mit Nährstoffen versorgt werden und diverse Infektionskrankheiten durchmachen.

\section{Nahrungsaufnahme von Menschen und Gemeinden, die mit HIV/AIDS leben}

Im Hinblick auf die Ernährungsunsicherheit und den biologischen Stoffwechsel bestehen im Zusammenhang mit der Nahrungsaufnahme diverse Probleme. Die Ergebnisse der Erhebungen zur Angemessenheit der Ernährung sind je nach Umfeld unterschiedlich. So ergab beispielsweise die quantitative Bewertung von Ernährungstagebüchern, die über drei Tage geführt wurden, bei HIV-positiven und HIV-negativen intravenös Drogenabhängigen in Boston, Massachusetts, USA, eine im Allgemeinen akzeptable Versorgung mit Makro- und Mikronährstoffen; wobei zwischen den beiden Untergruppen keine Unterschiede festgestellt wurden [77]. Eine gleich hohe Aufnahme reicht jedoch möglicherweise nicht aus, da ein unterschiedlich hoher Nährstoffbedarf belegt ist. Laut WHO [73] benötigen HIV-positive Menschen 10\% mehr Energie als HIVnegative Menschen, um ihr Gewicht zu halten und körperlich aktiv zu bleiben. Erwachsene mit klinischen Symptomen der AIDS-Erkrankungen benötigen sogar 20-30\% mehr Energie, um ihr Gewicht zu halten. Der geringere Body-Mass-Index der HIV-positiven Gruppe wurde eher dem durch die Erkrankung erhöhten Stoffwechselbedarf als den beschränkenden Faktoren in der Ernährung zugeschrieben [77]. Schätzungen gehen davon aus, dass Kinder, um einmal verlorenes Gewicht wieder zuzunehmen, einen um 50 bis 100\% erhöhten Energiebedarf haben [73].

Eine neue, von HIV Medicine herausgegebene Studie ergab ferner, dass Patienten, die bei Aufnahme der antiretroviralen Therapie unterernährt waren, erheblich schlechtere Überle- 
benschancen hatten. Unterernährte Patienten hatten eine um das Sechsfache höhere Mortalitätsrate als Patienten mit gutem Ernährungszustand [78]. HIV und Unterernährung bilden einen Teufelskreis, der das Immunsystem und den Körper durch wiederholte Infektionen schwächt.

Die Nährstoffaufnahme von HIV-infizierten Patienten ist jedoch nicht frei von paradoxen Aspekten. Kim et al. [79] untersuchten eine US-Population HIV-infizierter Erwachsener. Dreiundzwanzig Prozent dieser Patienten hielten Diät, mit dem Ziel, Gewicht abzunehmen. Menschen anderer Hautfarbe und intravenös Drogenabhängige nahmen weniger Energie zu sich als Weisse oder Menschen, die keine intravenösen Drogen konsumierten. Ein beträchtlicher Teil der Studienkohorte (52\%) nahmen nicht genügend Vitamin A und 11\% nahmen nicht genügend Eiweiss auf. Es ist klar, dass die derzeitige Literatur zur Nährstoffaufnahme sich auf die Industrienationen konzentriert und dass aus den Entwicklungsländern dringend mehr empirische Werte benötigt werden.

\section{Mythen und paradoxe Aspekte in der Beziehung zwischen Ernährungsunsicherheit und HIV/AIDS}

Rund um das Thema der Ernährungsunsicherheit und HIV/AIDS existieren eine Reihe von Mythen und paradoxen Aspekten. Wichtig ist, dass diese erkannt werden und dass man verhindert, dass diese Mythen und paradoxen Aspekte sich störend auf Politik bzw. Programme auswirken.

\section{Mythen ansprechen}

Konfliktsituationen, wie z.B. in der Elfenbeinküste, wirken sich nachweislich störend auf das Gesundheitswesen und die Bemühungen zur Prävention der HIV-Übertragung und zur Pflege und Betreuung infizierter Personen aus [80]. Wie aber wirkt sich die Nahrungsmittelunsicherheit auf die Übertragung der Krankheit aus? In einer Analyse erklärt der Hohe Kommissar der Vereinten Nationen für Flüchtlinge, Spiegel [81], dass Konflikt, Vertreibung, Nahrungsmittelunsicherheit und Armut die betroffenen Populationen anfälliger für eine HIV-Übertragung machten, sich aber die allgemeine Annahme, diese Anfälligkeit schlage sich unweigerlich in einer höheren Zahl an HIV-Infektionen nieder und heize folglich die HIV/AIDS-Epidemie an, nicht durch Daten belegen lasse. Wenn dies stimmt, dann ist die Lösung der in diesem Übersichtsartikel behandelten, mit der Ernährungsunsicherheit zusammenhängenden Probleme ganz klar palliativer Natur und lindert zwar das Leiden der Infizierten, trägt aber nicht dazu bei, die weitere Übertragung des Virus einzudämmen.

\section{Das Ringen mit paradoxen Aspekten}

Die Ernährungsunsicherheit auf Haushaltsebene kann wie bereits erwähnt mit Adipositas in Zusammenhang stehen. Ebenso können bestimmte Umwelt- und Verhaltensfaktoren sowie bestimmte Arzneimittel Übergewicht begünstigen, obwohl AIDS als «slim disease» (Abmagerungskrankheit) gilt. In den städtischen Regionen Afrikas entwickelt sich das Übergewicht möglicherweise zum Kontext für die HIV/AIDS-Ausbreitung. Die Transition and Health of South Africans-Studie ergab, dass die Urbanisierung in der nordwestlichen Provinz Südafrikas mit einer besseren Mikronährstoffaufnahme und einem besseren Mikronährstoffstatus, aber auch mit einem Anstieg an Übergewicht, Adipositas und diversen Risikofaktoren für nicht übertragbare Krankheiten einherging [82]. Das vermehrte Auftreten nicht ansteckender Erkrankungen bei schwarzen Südafrikanern in Verbindung mit der HIV/AIDSPandemie zeichnet für Mitarbeiter des Gesundheitswesens und Politiker ein komplexes Bild [83].

Massgeblicher Faktor hierfür ist, dass inzwischen auch arme Menschen mit Anti-HIV-Medikamenten behandelt werden. Die Nebenwirkungen bestimmter Therapien zur Behandlung der HIV-Infektion und der damit zusammenhängenden Infektionskrankheiten erhöhen das Risiko für Diabetes, Adipositas und das metabolische Syndrom; insbesondere im Hinblick auf das Risiko für kardiovaskuläre Erkrankungen gilt es daher, die Therapien sorgsam auszuwählen [84]. Forscher in Philadelphia, USA [85], stellen folgende interessante Verbindung her: (1) Adipositas macht Menschen anfällig für Diabetes und kardiovaskuläre Erkrankungen, und (2) dies sind genau die Komplikationen, die auch mit HIV, der Anti-HIV-Therapie bzw. der Kombination aus HIV und Anti-HIV-Therapie in Verbindung gebracht werden. Die Forscher stellten in ihrer klinischen Population in Philadelphia fest, dass im Zeitalter der Anti-HIV-Therapie mehr Patienten adipös (14\%) und übergewichtig (31\%) als abgemagert (9\%) waren [85]. In der Untergruppe der Frauen betrug die Adipositas-Prävalenz 28\%. In einer Gruppe von HIV-Patienten in Sao Paulo, Brasilien, die mit einer hochaktiven antiretroviralen Therapie behandelt wurden, um die HIV-Infektion in Schach zu halten, betrug die Prävalenz der zentralen Adipositas 45,7\%. Die Therapie wurde mit einer höheren Lipidaufnahme assoziiert, wobei sich das Risiko der zentralen Adipositas mit jeder um $10 \mathrm{~g}$ höheren Lipidaufnahme um das 1,3-fache erhöhte [86].

\section{Reaktionen der Politik und der Ernährungsprogramme auf die Ernährungsunsicherheit von Menschen, die mit AIDS/HIV leben, ihren Familien und Gemeinden}

In ihrem berühmten, 2003 in der Zeitschrift Science veröffentlichten Artikel stellen Rosegrant und Cline [87] fest, dass die weltweite Nahrungsmittelsicherheit auch in den nächsten 50 Jahren und darüber hinaus eines der globalen Probleme bleiben wird. Aufgrund geringerer Investitionen in Forschung und Infrastruktur sowie zunehmender Wasserknappheit sind die Ernteerträge in den letzten Jahren in vielen Regionen zurückgegangen. Die Klimaänderung und HIV/AIDS sind ebenfalls wichtige Faktoren, die sich in vielen Regionen entscheidend auf die Ernährungssicherheit auswirken. Die WHO-Beratung über den Nährstoffbedarf von Menschen, die mit HIV/AIDS leben, betonte, dass eine Rückbesinnung auf die Nahrungsressourcen und ihre Verwendung dringend erforderlich sei, da dies einen fundamentalen Teil der umfassenden Versorgung auf Landesebene darstelle [74]. Die Ernährungssicherheit verringert sich, wenn die zugrundeliegenden Nahrungssysteme unter Druck geraten [88]. Zu den Aspekten, die das System aus dem Gleichgewicht bringen, zählen die sich ändernden Faktoren in der 
Umgebung, wie z.B. Konfliktsituationen und HIV/AIDS. Die Folgen dieser Faktoren sind besonders schlimm, wenn sie in Kombination auftreten, wie dies in den letzten zehn Jahren z.B. im südlichen Afrika der Fall war. Daher müssen die Aspekte der Ernährungsunsicherheit im Kontext der HIV/AIDSPandemie auf globaler und nationaler Ebene sowie auf Ebene der Gemeinden und der Haushalts- bzw. der persönlichen Ebene betrachtet werden.

\section{Allgemeine Überlegungen der Grundsätze zur Linderung} der Ernährungsunsicherheit

Obwohl zunehmend evidenzbasierte Leitlinien und Programme erwartet werden, deren Wirksamkeit, Verträglichkeit und Wirtschaftlichkeit belegt ist, ergab die Suche nach den diesbezüglichen Belegen noch kein allgemein zufriedenstellendes Ergebnis. Laut dem vom Innenministerium der USA veröffentlichten Bericht gibt es trotz der steigenden Zahl an Interventionen bezüglich der Ernährungsunsicherheit in Gebieten mit hoher HIV/AIDS-Prävalenz nur wenige empirische Belege dafür, dass diese Programme die ernährungsbezogenen bzw. gesundheitlichen Outcomes oder die nachhaltige Verfügbarkeit von Nahrungsmitteln und den nachhaltigen Zugang zu diesen Nahrungsmitteln seitens der Zielpopulationen tatsächlich wirksam verbessern [14]. Angesichts des Kontexts und der gesammelten Erfahrungen kommt man jedoch schnell zu der Überzeugung, dass die Suche nach allgemein gültigen Massnahmen möglicherweise sinnlos sein könnte und dass die meisten Leitlinien zu HIV/AIDS ähnlich wie die Politik lokaler Natur sind.

Gillespie [89] hat die drei übergeordneten Leitprinzipien einer umfassenden Strategie zur Bekämpfung der Probleme der Ernährungsunsicherheit zusammengefasst, die sich im Zusammenhang mit HIV/AIDS ergeben. Dazu zählen: (1) die Stärkung der Widerstandskraft gegenüber HIV und AIDS auf Ebene der Haushalte und Gemeinden; (2) die nachhaltige Sicherung und Verbesserung des Lebensunterhalts in den betroffenen Gemeinden; und (3) die Gewährleistung eines entsprechenden sozialen Schutzes. Er warnt jedoch davor, dass diese Mobilmachung der Kräfte nicht separat und losgelöst, speziell für die HIV/AIDS-Krise, sondern eher in Form von Gesamtlösungen für die Gemeinden erfolgen sollte. Saasa [60] bietet eine ähnliche Querschnittsphilosophie und ruft zur Lösung der Probleme auf, die, insbesondere in Subsahara-Afrika, ursächlich für die Armut und stagnierende Entwicklung in den ländlichen Regionen der betroffenen Länder sind.

Laut Gillespie et al. [71] müssen die Leitsätze einer solchen Politik u.a. die Ressourcen anvisieren, auf den Umfang und die Grössenordung achten, eine Zusammenarbeit mit den entsprechenden Partnern vorsehen, zusätzliche Notfälle einplanen und diesbezügliche Programme entwickeln sowie Überwachung und Bewertung berücksichtigen. Bei den spezifischen Interventionen zur Verbesserung der Ernährungssicherheit im Rahmen der HIV/AIDS-Epidemie liegen die Prioritäten, die es zu verfolgen gibt, in der gezielten Stärkung der folgenden Bereiche: der Landwirtschaft, des Gartenbaus auf Haushaltsund Gemeindeebene, der Nahrungsmittelhilfe, der Wasserversorgung, der Hygiene und Umweltgesundheit sowie des Managements der natürlichen Ressourcen der Gemeinde [90, 91].
Dementsprechend dienen konzertierte Aktionen zur Bekämpfung der in den von HIV/AIDS betroffenen Gesellschaften herrschenden Armut und Ungerechtigkeit auch dazu, die Auswirkungen der Infektion zu bekämpfen. Gillespie [90, 91] bezeichnet dies als «HIV/AIDS-Mainstreaming», ein Ansatz, bei dem die Bekämpfung von HIV/AIDS zu einem Teil der Massnahmen wird, die zur Lösung der in der Gemeinde herrschenden Probleme ergriffen werden. Dies ist eine der Taktiken, bei der wirkungsvolle Massnahmen ergriffen werden können, ohne dass es zu einer Stigmatisierung der Begünstigten kommt. Fortschritte werden erzielt, indem die entsprechenden Kapazitäten bereitgestellt und vernünftige Anreize zur Nutzung dieser Kapazitäten geboten werden [90, 91].

\section{Landwirtschaftliche Hilfe und Entwicklung zur Linderung} der Ernährungsunsicherheit

Zur landwirtschaftlichen Intervention zählt im weitesten Sinne der Gartenbau auf Haushalts- und Gemeindeebene sowie der Anbau von Getreide und die Tierzucht, um für Einkommen und Nahrung zu sorgen. Der Gartenbau konzentriert sich darauf, für Nahrungsvielfalt und die Anreicherung der Nahrung mit essentiellen Mikronährstoffen zu sorgen. Da die Gemeinden möglicherweise keine Erfahrung im Gartenbau besitzen, muss man sich bemühen, den Gemeinden die Fertigkeiten zur Anlage eines Gartens zu vermitteln und Verhaltensänderungen herbeizuführen, um die Aufmerksamkeit darauf zu lenken, dass die nährstoffreichen, lokal erzeugten Nahrungsmittel unbedingt verzehrt werden müssen.

Die Subsistenzwirtschaft lässt sich dauerhaft sichern, wenn Nutzpflanzen, die nur minimale Pflege brauchen, bzw. arbeitssparende Massnahmen, wie z.B. das Umpflügen der Anbauflächen durch Tiere, eingeführt werden und traditionelle Anbaumethoden ersetzen [71]. Die Diversifizierung der Produktion und die Einführung landwirtschaftlicher Technologien, die den Einsatz von Arbeitskräften oder diesbezügliche Engpässe reduzieren (z.B. kein Pflügen), tragen ferner zu einer geringeren Belastung des Humankapitals bei. Die Arbeitsbelastung lässt sich ebenfalls durch Einführung entsprechender Technologien senken, z.B. kleine und arbeitssparende Technologien zur Nahrungsverarbeitung, treibstoffsparende Öfen und Wasserpumpen. Parley et al. fördern die Verbesserung und Anpassung der landwirtschaftlichen Systeme durch eine dem jeweiligen Hintergrund entsprechende Forschung und Entwicklung [92], mit dem Ziel, die Länder autarker und die Gemeinden widerstandsfähiger zu machen. Sie rufen zu Investitionen in neue, für die Nahrungsproduktion in Entwicklungsländern geeignete Technologien auf. Auch die im Bereich der Mikrofinanzen angebotenen Optionen können genutzt werden, um für die Betriebskosten von Zugtieren, eingesetzte Produktionsmittel, Leiharbeiter etc. aufzukommen. Die Gemeinden sollten ferner ermutigt werden, ihr praktisches Wissen und ihre Erfahrung auf dem Gebiet der Landwirtschaft mit den Witwen, Waisen und anderen Überlebenden zu teilen und u.a. die landwirtschaftliche Ausbildung in die Lehrpläne der Schulen zu integrieren. 
Tabelle 4. Optionen des Welternährungsprogramms bezüglich des Einsatzes der Nahrungsmittelhilfe zur Linderung von HIV/AIDS

1 'Food for Vocational Training'-Programme für Strassen- und Waisenkinder, bei denen die Kinder im Gegenzug für eine Berufsausbildung Nahrungsmittel erhalten

2 Schulspeisung und Lebensmittelrationen zum Mitnehmen für Familien, die für Waisenkinder sorgen

3 'Food for Training'-Programme, die Erwerbstätigkeiten (Pilzzucht, Knüpfbatik, etc.) fördern und mit Mikrokrediten für Frauen und Waisen einhergehen

4 'Food for Training'- und 'Food for Work'-Programme, mittels derer Landwirte durch Zugtiere und die Bereitstellung von Saatgut und landwirtschaftlichen Werkzeugen unterstützt werden

5 'Food for Work'-Programme zur Steigerung der landwirtschaftlichen Produktion durch privaten Gartenbau, um so für eine abwechslungsreichere Ernährung und eine verbesserte Mikronährstoffaufnahme zu sorgen

6 'Food for Work'- und 'Food for Training'-Programme zur Förderung der nicht arbeitsaufwändigen Tierhaltung im kleinen Umfang, um für (a) eine höhere Aufnahme energie- und proteinreicher Nahrungsmittel und für (b) Kapital und Ersparnisse zu sorgen, die im Laufe der Zeit anwachsen.

In abgeänderter Form nach Anpassung aus dem Welternährungsprogramm [94] übernommen von Gillespie et al. [71].

\section{Direkte Nahrungsmittelhilfe zur Linderung der}

Ernährungsunsicherheit

Hauptargument für die Nahrungsmittelhilfe sind die humanitären Grundsätze der Versorgung unterernährter Menschen mit Nahrungsmitteln. In Regionen mit einer knappen Versorgung an antiretroviraler Therapie liesse sich mit einer über die Ernährung gesicherten, ausreichenden Nährstoffaufnahme ein eindeutiger palliativer Nutzen erzielen. Dies fassen auch Tabi und Vogel wie folgt zusammen [43]: «Der Gesundheits- und Ernährungsstatus der Patienten lässt sich mittels nahrhafter Nahrung verbessern und den Patienten kann somit ein längeres und hochwertigeres Leben ermöglicht werden.» Man geht davon aus, dass die Ernährungshilfe das Leben von Patienten verlängern kann und dass dies sowohl den Patienten selbst als auch den Menschen nützt, die hinsichtlich der Betreuung und Versorgung von den Patienten abhängig sind [71].

In der Entwicklungshilfe gilt die direkte Nahrungsmittelhilfe bei manchen Menschen seit langem als Tabu, da sie deren Ansicht nach tendenziell Abhängigkeiten erzeugt und die landwirtschaftlichen Märkte stört. Tatsächlich aber hat HIV/AIDS in armen Ländern bereits eine solche Abhängigkeit erzeugt und eine Störung der Märkte verursacht. Es stellt sich daher die Frage, wie sich die Nahrungsmittelhilfe am besten einsetzen lässt, um die Auswirkungen der Epidemie zu lindern. Kadiyala und Gillespie [93] haben vor Kurzem die Möglichkeiten der Nahrungsmittelhilfe im Einzelnen überprüft und sie in erster Linie als soziales Sicherheitsnetz betrachtet. Laut US-Innenministerium können sich die Interventionen der Nahrungsmittelhilfe positiv auf das Leben der Empfänger auswirken, da sie deren Abhängigkeit von gefährlichen Bewältigungsstrategien, die den
Lebensunterhalt untergraben, verringert, für eine vielseitigere und qualitativ hochwertigere Ernährung sorgt und die Ernährungssicherheit auf Haushaltsebene verbessert [14]. Rukuni [59] äussert gegenüber den Regierungen ähnliche Denkanstösse, um diese zu weiteren Strategien der Nahrungsmittelsicherheit zu veranlassen, die den vulnerablen Gruppen einen besseren $\mathrm{Zu}$ gang zu Nahrungsmitteln bieten.

Ist die direkte Nahrungsmittelhilfe tatsächlich ein nützlicher Bestandteil des Sicherheitsnetzes, so kommen bei ihrer Umsetzung sowohl Vorbehalte als auch Kreativität ins Spiel. Im Allgemeinen sollte die Nahrungsmittelhilfe in einem Land auf die Regionen abzielen, in denen die Versorgung mit Nahrungsmitteln am unsichersten ist und in denen die Haushalte nicht in der Lage sind, ihren Nahrungsbedarf zu decken [71]. Die organisierte Nahrungsmittelhilfe sollte ferner die Bewältigungsund Entwicklungsstrategien der Haushalte und Gemeinden nicht unterminieren [71]. In diesem früheren Übersichtsartikel [71] wurden die Ideen des Welternährungsprogramms [94], die Nahrungsmittelhilfe einzusetzen, um bestimmte Ausbildungsund Produktivitätsziele (Tabelle 4) zu erreichen und gleichzeitig die Ernährung unterernährter Menschen zu unterstützen, vor dem Hintergrund der HIV-Epidemie untersucht. Kadiyala und Gillespie [93] betrachteten jüngst das Thema Nahrungsmittelhilfe in dem etwas weiter gefassten Zusammenhang der Sicherung des Lebensunterhalts. Anscheinend besteht immer mehr Bereitschaft zur Erforschung der zunehmend kreativen Einsatzmöglichkeiten der Nahrungsmittelhilfe. Gemeinden sind komplexe soziale Gebilde; stellt die Ursache für die Notwendigkeit der Nahrungsmittelhilfe einen Grund zur Stigmatisierung dar, was bei einer HIV-Infektion im Allgemeinen zutrifft, so besteht eine der Vorsichtsmassnahmen darin, die Leistungsempfänger nicht offen $\mathrm{zu}$ nennen [95]. Letztendlich jedoch gibt es immer wieder Menschen, die stetig die alte Leier wiederholen, dass Nahrungsmittelhilfe auch stets eine «Ausstiegsstrategie» umfassen müsse, um permanente Abhängigkeit zu vermeiden [14].

\section{Die Grundsätze von Interventionsprogrammen auf \\ lokaler Ebene}

Der begrenzte Umfang der bestehenden Programme ist Gegenstand ständiger Kritik und kritischer Äusserungen, wie zum Beispiel, dass die von Organisationen durchgeführten Interventionen zur Verbesserung der Ernährungssicherheit in Regionen, in denen HIV/AIDS endemisch ist, in der Tendenz nur vereinzelte, kleine Projekte seien, deren Ergebnisse nicht verallgemeinert werden könnten [14]. Vielleicht werden manche Projekte nur in einem kleinen Massstab angelegt, um der klugen Leitlinie zu folgen, dass sich Interventionen auf die Regionen konzentrieren sollten, in denen die Ernährungsunsicherheit am grössten ist $[8,71]$. Es handelt sich um Einzelprojekte, weil die am stärksten betroffenen Gemeinden sowohl aufgrund ihrer geographischen Abgeschiedenheit als auch aufgrund ihrer sozialen Stigmatisierung isoliert sind. Gelten jedoch die Grundsätze, dass die Gemeinden $\mathrm{zu}$ eigenverantwortlichem Handeln ermächtigt werden sollen, wird es sich bei den Interventionen nicht um «strenge Protokolle» handeln, da die Gemeinden im Rahmen der jeweils herrschenden Umstände ihren eigenen Weg fin- 
den müssen und diese Umstände gleichzeitig Änderungen unterworfen sind.

Rosegrant und Cline betonen in ihrer Abhandlung zur globalen Ernährungssicherheit [87], dass das fundamentale Recht der Gemeinden, an allen Schritten der Programmentwicklung beteiligt zu sein, für eine gute Demokratie von elementarer Bedeutung ist. Auch Rutengwe betont, dass es wichtig sei, lokale Programme zu entwickeln, in denen den besonderen Schwierigkeiten einer Gemeinde zum Zwecke der praktischen Forschung durch Massnahmen Rechnung getragen wird [8]. Die Studie ergab, dass das Wechselspiel zwischen der chronischen Ernährungsunsicherheit in städtischen Haushalten, der Prävalenz der Unterernährung und der Einkommensarmut das grösste Problem darstellte. Ferner zeigte die Studie, dass ein interaktiver Ansatz, beispielsweise mittels eines Problem- und eines Lösungsbaums, nützlich ist, um die Ursachen und Wirkungen der in einem bestimmten Gebiet vorliegenden Situation logisch zu analysieren und lokale Massnahmen mit maximalem Einsatz der Beteiligten auf Gemeindeebene zu formulieren.

\section{Eingehende Suche nach Lösungen auf dem}

Afrika-Forum 2006

Die theoretischen Grundsätze und akademischen Erkenntnisse bieten keine praktischen Lösungen für die mit der Ernährungsunsicherheit konfrontierten Gemeinden und Personen. Ein Beispiel für aktuelle pragmatische Massnahmen, die ergriffen werden, um über einen Erfahrungsaustausch mit den an vorderster Front tätigen Ärzten aus der Praxis zu lernen, ist die von der Hilfsorganisation Project Concern International einberufene und im Mai 2006 in Lusaka, Sambia, abgehaltene Konferenz. Unter dem Motto «Das Afrika Forum 2006: ein ganzheitlicher Ansatz zur Bekämpfung der HIV/ AIDS-Epidemie und der Ernährungsunsicherheit» [96] versuchte das Forum, den Teilnehmern die Möglichkeit zu geben, ihre Erfahrungen mit den schwierigen Problemen der Epidemie und mit der Entwicklung und der Umsetzung ganzheitlicher Programme direkt auszutauschen. Die Teilnehmer tauschten im Laufe der Woche ihre Fachkenntnisse aus und eigneten sich praktische Lösungen an, um die Nahrungsversorgung und den Lebensunterhalt in ihren eigenen Gemeinden besser abzusichern. Ferner legten die Teilnehmer auch alle Prioritäten und Änderungen fest, die in allen Phasen der Entwicklung eines ganzheitlichen Programms zu den Themen HIV/AIDS und Ernährungssicherheit notwendig sind. Diese Gedanken und Praxiserfahrungen wurden in der Erklärung des Afrika-Forums 2006 festgehalten.

Das Forum versuchte, die Fertigkeiten der in der Praxis tätigen Mitarbeiter rund um die Entwicklung ganzheitlicher Programme zu stärken, indem es das Lernen von Kollegen erleichterte, und die praktischen Ärzte anhand konkreter Beispiele, in denen Gemeinden erfolgreich auf die Herausforderungen der HIV/AIDS-Epidemie und der Ernährungsunsicherheit reagierten, zu motivieren und zu befähigen. Der Lernprozess basierte auf den Prinzipien der Erwachsenenbildung und der Verhaltensänderung. Um bei Studierenden im Erwachsenenalter einen Lernprozess zu erzielen, der auf bereits vorhandenem Wissen und Kenntnissen aufbaut, müssen die Studierenden aktiv in den Lernprozess eingebunden werden.

HIV, Ernährungssicherheit und Gesundheit von Kindern
Dies gilt insbesondere für die tatkräftigen, in der Praxis tätigen Menschen, die einen dynamischen und praktischen Lernansatz benötigen, der sich an den Fertigkeiten orientiert. Beim Afrika-Forum musste jeder Teilnehmer sowohl als Studierender teilnehmen als auch selbst aktiv Beiträge liefern. Das bedeutete, dass jeder Teilnehmer in der einen oder anderen Form anwesend sein und aktiv an den praktischen Tätigkeiten teilnehmen musste, die das Wissen zu einem bestimmten Aspekt der Entwicklung ganzheitlicher Programme bezüglich HIV/ AIDS bzw. Nahrungsmittel- und Ernährungssicherung vermittelten. So mussten die Teilnehmer beispielsweise selbst Gärten umgraben und anlegen, Nahrung zubereiten und Gemeindeprojekte besichtigen.

Das Forum [96] sprach die praktischen Probleme an, mit denen sich die Berater z.B. bei der Umsetzung der Best Practices in der Säuglings- und Kleinkindernährung konfrontiert sehen. Die Einhaltung der Ernährungsprotokolle, die ein ausschliessliches Stillen in der Anfangsphase gefolgt von einem schnellen Abstillen vorsehen, werden insbesondere durch Stigmatisierung und Kosten behindert. In Sambia wurden Gemeindegärten angelegt, um die Qualität der Ernährung, wie von Gopalan betont, über eine reine Deckung des Energiebedarfs hinaus zu verbessern [47]. Beim Afrika-Forum wurden eine Reihe von Gartenbauprojekten vorgestellt, welche die vielen Möglichkeiten der Nahrungsmittelsicherheit, der verbesserten Ernährung und eines verbesserten Auskommens erläutern. Ein Beispiel für diesen ganzheitlich-synergetischen Ansatz ist ein in Malawi durchgeführtes Projekt mit dem Titel «Verbesserte Existenz durch bessere Nahrung», das die Agenda für Ernährungssicherheit in den von HIV betroffenen Gemeinden mit Umwelt-, Bildungs- und Gesundheitskomponenten auf Haushaltsebene kombiniert [96]. Bezüglich der Ausdehnung des ganzheitlichen Konzepts auf eine Synergie mit ersten Initiativen der Drogentherapie herrschte beim Afrika-Forum Übereinstimmung, dass die Initiativen der Drogentherapie ohne eine ausreichende Menge an Nahrungsmitteln entsprechender Qualität nicht wirklich lebensverlängernd wirken [96].

Die Landwirtschaft selbst wurde im Forum [96] in einem ganzheitlichen Zusammenhang betrachtet, und Erfahrungen mit der Erzeugung landwirtschaftlicher Produkte zur Erzielung von Einkommen wurden ausgetauscht. In Sambia hatten sich Witwengruppen zusammengeschlossen, um Pilze zu züchten, die sie in den Handel brachten, um so die Ernährungssicherheit des Haushalts zu erhöhen. Zu den weiteren Erwerbstätigkeiten, die auf dem Forum vorgestellt wurden, zählten die Bienenhaltung zur Erzeugung von Honig und die Tierhaltung.

Nicht alle Probleme der Nahrungsmittelsicherheit lassen sich auf landwirtschaftlicher Ebene lösen. Die Sicherheitsnetze der Regierungs- und Nichtregierungsorganisationen, die u.a. die Verteilung von Finanz- und Nahrungsmitteln an Familien umfassen, die um ihre Existenz kämpfen, sind Teil einer ganzheitlichen Lösung. Marchione [95] hat, wie bereits erwähnt, die Entwicklung von Programmen gefordert, welche die Probleme der konkreten Hilfestellungen auf Gemeindeebene umgehen, um sowohl eine eventuelle Stigmatisierung der Empfänger der Hilfsleistungen als auch die Entstehung von Missgunst in den Teilen der Bevölkerung, die keinen Anspruch auf diese Hilfsleistungen haben, zu vermeiden. 


\section{Betreuung und Pflege der kindlichen Gesundheit}

\section{Die Betreuung und Versorgung von Kindern und traditionelle Gesundheitspraktiken}

Die Gesundheit von Kindern, und zwar sowohl von HIVpositiven als auch HIV-negativen Kindern, von Waisenkindern und Kindern, die bei ihren Eltern leben, hängt nicht nur von der Nahrungsmittel- und Ernährungssicherheit $\mathrm{ab}$, sondern auch von der Qualität der Betreuung und Versorgung, die ihnen zuteil wird. Diese wiederum hängt von den in der Gemeinde und dem Haushalt verfügbaren Ressourcen ab (u.a. der Energie und der Zeit) sowie von den Versorgungsmethoden und den Entscheidungen der Betreuer. Je ärmer das Umfeld, desto wichtiger wird die Versorgung für das Überleben, das Wachstum und die Entwicklung der Kinder.

Das Rahmenkonzept von UNICEF für die Ernährung wurde 1990 erarbeitet und 1992 auf der Internationalen Ernährungskonferenz in Rom eingeführt. Laut diesem Rahmenkonzept sind drei Faktoren massgeblich für das Überleben, das Wachstum und die Entwicklung von Kindern [97, 98]. Zwei dieser Faktoren sind die Ernährungssicherheit auf Haushaltsebene sowie die Gesundheitsversorgung und die Umwelt. Das dritte Element in der Triade ist die Betreuung, die wie folgt definiert wird: "Die auf Haushalts- und Gemeindeebene aufgewendete Zeit, Aufmerksamkeit und Unterstützung zur Gewährleistung des körperlichen, geistigen und sozialen Wohlbefindens des heranwachsenden Kindes und anderer Haushaltsmitglieder.» Laut der verfeinerten Theorie von Martin-Prevel [97] unterscheidet man bei den Betreuungsressourcen auf Haushaltsebene drei Kategorien: die humanen Ressourcen (Wissen, Anschauungen, Bildung, physische und mentale Gesundheit der Betreuer); die wirtschaftlichen Ressourcen (Steuerung von Einkommen, Arbeitspensum und Zeit) und die organisatorischen Ressourcen (wechselnde Betreuer, Unterstützung in der Gemeinde).

Martin-Prevel [97] hat die konkreten Elemente der Betreuung zerlegt und priorisiert. Gemäss Tabelle 5 unterscheidet man sechs Kategorien der Betreuung. Im Allgemeinen geht man davon aus, dass die Kinder in erster Linie von der Mutter betreut werden. Es ist klar ersichtlich, dass alle sechs Kategorien im Zeitalter von HIV/AIDS für die Gesundheit der Kinder von grösster Bedeutung sind. Die oben genannten Praktiken der Betreuung zielen zuerst auf den Schutz der Betreuer ab, sorgen weiter für die Ernährung der Familie und dann für eine Abschwächung der gesundheitsschädlichen Faktoren in der Umwelt.

Zu den Gebieten, in denen sich die HIV/AIDS-Epidemie mit am schnellsten ausbreitet, zählen die städtischen Gebiete des indischen Subkontinents. Wichtig ist in diesem Zusammenhang, dass Kinder, die in Slums aufwachsen, von allen städtischen Gruppen den schlechtesten Ernährungsstatus aufweisen, der sogar schlechter ist als der durchschnittliche Ernährungsstatus der auf dem Land lebenden Kinder [99]. Das Fehlen der richtigen Betreuung der Kinder verschlimmert diese Situation weiter. Kinder aus armen Familien sind, ähnlich wie bei der Ernährungssicherheit, anfälliger für gefährliche Praktiken in der Kinderbetreuung und die zusätzliche
Tabelle 5. Die sechs Kategorien der Betreuung

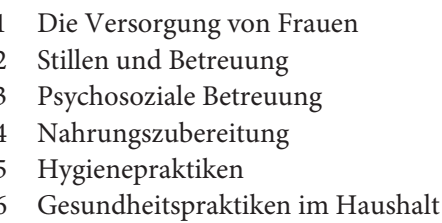

Nach Martin-Prevel [97].

Last der AIDS-Epidemie stellt eine starke Belastung der ohnehin schon schwierigen Situation dar.

\section{Waisen und Betreuung ausserhalb der Familie}

Die Verwaisung ist eine verhängnisvolle Folge der unter den Erwachsenen zunehmenden HIV/AIDS-Epidemie. Sie stellt in einer Gemeinde einen der grössten Risikofaktoren für einen schlechten Gesundheitszustand und Mangelernährung bei Kindern dar. Shetty und Powell [100] bemerkten hinsichtlich der HIV/AIDS-bedingten Verwaisung, dass die durch die HIV/AIDS-Pandemie verursachte Waisenkrise international nach wie vor ein ernstes Problem mit langfristigen sozialen Auswirkungen darstelle. Ende 2001 hatten schätzungsweise 14 Millionen Kinder weltweit ihre Mutter oder beide Elternteile aufgrund von AIDS oder aus AIDS-bedingten Gründen verloren. Am stärksten betroffen ist das südliche Afrika, wo mehr als $80 \%$ der AIDS-Waisen leben. Ohne die Versorgung seitens der Eltern oder eines ernannten Betreuers wird das Risiko dieser Kinder für Mangelernährung, einen schlechten Gesundheitszustand, eine unzureichende Schulbildung, Migration, Obdachlosigkeit und Missbrauch wahrscheinlich aussergewöhnlich hoch sein [100].

Laut Schätzungen beläuft sich die Zahl der primär AIDSbedingten Waisen in Uganda derzeit auf zwei Millionen [101]. Es wird angeführt, dass die sich im nördlichen Teil des Landes bezüglich der Versorgung dieser Waisen ergebenden Änderungen geschichtlich bedingt sein müssen, wobei vor allem die Auswirkungen bewaffneter Konflikte und die seit den späten 70er Jahren des 20. Jahrhunderts stattgefundene Entwurzelung des lokalen Hirtentums und der auf Baumwolle basierenden Wirtschaft eine Rolle spielten [101]. Diese Prozesse bedingten in Kombination eine dramatische wirtschaftliche Marginalisierung mit äusserst besorgniserregenden Folgen für Waisenkinder und ihre Betreuer. Nyambedha et al. [102] fanden in einer im ländlichen Kenia durchgeführten anthropologischen Studie heraus, dass die Haushalte, in denen Waisen leben, es trotz des vorherrschenden Mangels an Nahrung, medizinischer Versorgung und Kleidung als am schlimmsten empfanden, dass sie sich die Schulgebühren nicht leisten konnten. Obwohl die Ressourcen bei weitem nicht ausreichten, trugen die traditionellen, verwandtschaftsbasierten Hilfssysteme doch stark zur Versorgung der Waisenkinder bei. Laut Beobachtungen aus dem südlichen Afrika senkt bereits die reine Anwesenheit der Eltern bei Kindern das Risiko für Untergewicht von 39\% auf 21\%. Bei Kindern mit zwei lebenden Elternteilen betrug das Risiko für Untergewicht lediglich 10\% [12]. 
Die Reformierung der Einstellungen und der Methoden zur Versorgung und Betreuung von Kindern

Manchen erscheint es anmassend, dass westliche Fachleute aus wohlhabenden Industrieländern die Einstellungen und Methoden der Kinderpflege in nicht industrialisierten Gesellschaften mit niedrigen Einkommen kritisieren und zu «reformieren» versuchen [103]. Der Gipfel der Arroganz ist laut diesen Kritikern die Unterscheidung in "gute» traditionelle Methoden, die es zu erhalten gilt und «verbesserungswürdige» Methoden. Diese Kritiker fordern eine generelle Respektierung der traditionellen Vorgehensweisen, die sich laut ihrer Argumentation bewährt hätten, das Produkt einer langen gesellschaftlichen Entwicklung und Anpassung seien und die kulturelle Weisheit verkörperten. Wer seien schon UNICEFAnthropologen oder Akademiker, dass sie einer Gemeinde eine neue Agenda bezüglich der Versorgung und Betreuung auferlegen könnten.

Tatsächlich sind die traditionellen Methoden der Kinderpflege das Produkt der kulturellen Entwicklung der Gesellschaften, und die Regeln der Evolution somit massgeblich, um den Ursprung dieser Methoden und ihre Auswirkungen auf die kindliche Gesundheit zu verstehen. Laut der Theorie von Darwin ist es nicht das Ziel der Evolution, dass alle Lebewesen, die geboren werden, auch überleben. Die Landflächen, die den Nomadenstämmen zur Verfügung standen, konnten nur eine begrenzte Zahl an Jägern und Sammlern ernähren, so dass die Evolution für das Überleben der Stämme und somit für das Überleben der Spezies Mensch eher an einer gleichbleibenden als an einer wachsenden Zahl an Menschen interessiert war. Im Kampf um das Überleben waren natürlich die widerstandskräftigsten und die am besten an die jeweilige Umgebung mit ihren vielen Herausforderungen angepassten Nomaden für die Weiterführung der Erblinien des Stamms am wertvollsten.

Folgt man dieser Logik, so darf man nicht davon ausgehen, dass die traditionellen Methoden der Kinderpflege evolutionsbedingt zwangsläufig auf das Überleben aller Menschen ausgerichtet wurden. Dies hätte eine Überpopulation in den Territorien zur Folge gehabt. Tatsächlich ist es möglicherweise ein Kennzeichen der traditionellen Kinderpflege, dass sie die Kinder bezüglich des Überlebens vor bestimmte Anforderungen stellt, welche bei bestimmten Kindern die angeborene, natürliche Anpassung zur Meisterung der in der Umwelt auftretenden Gefahren erzeugt. Kinder, die diese Prüfungen nicht bestanden, machten gemäss dem klassischen Prinzip der Evolutionsbiologie «Survival of the Fittest» Platz für Kinder, die diese Anforderungen meisterten.

Soweit unsere moderne Sozialethik darauf beruht, dass wir das Recht aller Kinder auf Überleben und Gedeihen uneingeschränkt respektieren, muss ein eventueller evolutionärer Imperativ hinsichtlich der Betreuungs- und Versorgungsmethoden durch den humanitären Imperativ ersetzt werden, der tatsächlich das Überleben aller Lebewesen zur Maxime macht. Die Rechtfertigung zur Reformierung der traditionellen Betreuungs- und Versorgungsmethoden besteht genau in dieser Vergewisserung, dass Verhaltensweisen darauf ausgerichtet sind, die Gesundheit aller zu schützen. Im Idealfall benötigen HIV-infizierte Kinder möglicherweise eine andere Betreuung

HIV, Ernährungssicherheit und

Gesundheit von Kindern und Versorgung als nicht infizierte Kinder, um die Risiken in der Umwelt, die ihre Gesundheit bedrohen und ihr Überleben gefährden würden, noch weiter abzubauen.

Abgesehen von der endemischen Armut und der Ressourcenknappheit entsteht die Wechselwirkung zwischen Betreuer und den Pflege- und Versorgungspraktiken vor dem Hintergrund geeigneterer und häufig neuer, einzuführender Methoden. Mit Dezimierung der Elterngeneration müssen Kleinkinder häufig von älteren Geschwistern oder Grosseltern gepflegt und betreut werden. Letztere sind möglicherweise stärker in den traditionellen Methoden der Kinderpflege und -versorgung verwurzelt, die sich eher in Richtung eines selektiven als eines gesicherten Überlebens auswirken; eine Änderung der grosselterlichen Traditionen würde sich wahrscheinlich als eher schwierig erweisen. Die älteren Geschwister haben hinsichtlich der Pflege und Versorgung von Kindern nur minimale Erfahrung und wären sozusagen ein unbeschriebenes Blatt, das hinsichtlich der neuen Einstellungen und Verhaltensweisen beeinflusst werden könnte. Aber gerade ihr junges Alter und ihr Mangel an Erfahrung als Eltern nimmt ihnen das Selbstvertrauen für jüngere Geschwister zu sorgen. Somit wird die Pflege und Versorgung der Kinder zur Förderung eines guten Gesundheitsstatus in den Gebieten, in denen HIV endemisch ist, durch die Erkrankung bzw. die Abwesenheit der Eltern, auf eine höchst unsichere Grundlage gestellt.

Die Pflege und Versorgung der Kinder leidet ferner unter der übermässigen Belastung der menschlichen Arbeit durch HIV/AIDS. Gillespie et al. [6] bieten eine Analyse der weltweit durch HIV/AIDS verursachten Kosten. 1990 stand HIV/AIDS weltweit an 28. Stelle als Ursache für Lebensjahre, die aufgrund von frühzeitigem Tod verloren gehen oder von Behinderung geprägt sind (disability-adjusted life years, DALYs), und Hochrechnungen gehen davon aus, dass die Pandemie im Jahr 2020 an 10. Stelle stehen wird. Man vermutet, dass HIV/ AIDS im südlichen Afrika (vom siebten Platz im Jahr 1990) und in Indien (wo ein enormer Anstieg von einem niedrigen Platz stattgefunden hat) bis zum Jahr 2020 sogar an die dritte Stelle der Ursachen von DALYs rücken wird. Die Autoren erklären weiter, dass diese Zahlen die vollen Auswirkungen auf die menschliche Arbeit nicht in ihrer ganzen Tragweite wiedergeben, da sie die Arbeit von gesunden Menschen, die aufgrund von HIV/AIDS auf andere Aktivitäten, wie z.B. die Pflege infizierter und kranker Menschen, umgelenkt wird, nicht umfassen. White und Robinson [104] erklären, dass die ermittelten Auswirkungen auf die Zahl der Menschen, die pro Haushalt von einem Erwerbstätigen abhängig sind, nicht schlüssig seien. Ein Grund hierfür sind möglicherweise die Bewältigungsmechanismen der Gemeinden oder Familien, die Kinder u.a. über verwandtschaftliche oder andere inoffizielle Netzwerke in Pflegefamilien unterbringen, damit die Arbeitskräfte im besten Alter bei der Erzeugung von Nahrungsmitteln helfen können. Diese Arbeitsumverteilung führt möglicherweise dazu, dass Kinder eine schlechtere Pflege und Versorgung erhalten und einem erhöhten Risiko für eine HIV-Übertragung ausgesetzt sind. Die Versorgung der Kinder mit elementarer Bildung wird ebenfalls zunichte gemacht, da die Kinder aus der Schule genommen werden, um entweder bei der Krankenpflege zu helfen oder mit für das Auskommen der 
Familie zu sorgen. Letzteres bedeutet häufig die Teilnahme an riskanten Verhaltensweisen, die nach und nach zu einer weiteren HIV-Ausbreitung führen.

$\mathrm{Da}$ es unwahrscheinlich ist, dass in den bereits heute verarmten Gemeinden neue externe Versorgungsressourcen $\mathrm{zu}$ Tage treten, wurde ein Ansatz der positiven Abweichung (PA) zur Sprache gebracht [105-107] und erforscht. Bei einer Untersuchung der positiven Abweichung werden ungewöhnliche Verhaltensmuster seitens der Betreuer bzw. in den Haushalten, die bei den Kindern einer Gemeinde zu positiven Outcomes führen, identifiziert [106]. Diese Verhaltensmuster bezeichnet man auch als PA-Methoden. Man vermutet, dass Programme, die darauf abzielen, diese PA-Methoden bekannt zu machen, wahrscheinlich keinen Forschungszwängen unterliegen würden. Auf Gemeindeebene sollten diese PA-Programme u.a. die Planung, die Ausrichtung auf die Gemeinde, die Analyse der jeweils vorliegenden Situation, die Untersuchung der positiven Abweichung und das Feedback der Gemeinde zu den geplanten Massnahmen umfassen [106]. Ein Beispiel stammt aus den ländlichen Regionen Pakistans, wo Methoden der Neugeborenenpflege vom ausschliesslichen Stillen über den Wärmeschutz bis zur Hygiene als positiv abweichende Methoden identifiziert und in der Folge gefördert wurden [106]. Ein weiteres Beispiel stammt aus Nordvietnam und behandelt die Qualität des Fütterns von Kleinkindern [107]. Das Programm zeigte, dass ein positives und aufgeschlossenes Füttern mit entsprechenden verbalen Äusserungen und Ermutigungen bei Kindern im zweiten Lebensjahr genau richtig war, um die Kinder zur Nahrungsannahme zu bewegen und somit einen verbesserten Ernährungs- und Gesundheitsstatus der Kinder zur Folge hatte.

Es beginnt sich abzuzeichnen, dass die Pflegequalität und die Befähigung der Betreuer spezifisch und deutlich mit der HIV/AIDS-Epidemie verschmolzen sind [105, 107, 108]. Lapping et al. [105] machten im Zusammenhang mit dem in dieser Übersichtsarbeit behandelten Thema (d.h. HIV/AIDS und Ernährungssicherheit) frühzeitig Erfahrungen mit dem Ansatz der positiven Abweichung. Die Autorengruppe vermutet, dass ihre Intervention, die sich der positiven Abweichung bedient, schneller Änderungen herbeiführt, da sie die Mobilisierung der Gemeinde beschleunigt, das Bewusstsein erhöht, Probleme löst, zu Verhaltensänderungen motiviert sowie neue Verhaltensweisen empfiehlt und diese tatsächlich einführt. Die Wissenschaftler räumen jedoch ein, dass eine mögliche, auf den Ansatz der positiven Abweichung zurückzuführende Nutzwirkung hinsichtlich der Ernährungssicherheit und der gesundheitlichen Outcomes noch zu quantifizieren bleibt [107]. Aus Ostkenia, wo Familien, die für HIV-positive Kinder sorgen, vom Lea Toto Kangemi Outreach-Programm unterstützt werden, wurden eine Reihe von Erfahrungen veröffentlicht [108, 109]. Viele dieser Kinder sind Waisen oder stehen kurz davor Waisen zu werden. Die Studie ergab, dass in den Fällen, in denen Stigmatisierung keine Rolle spielte, ein grösseres Wissen über die richtige Pflege und Versorgung vorlag. Innerhalb der Gemeinden wurden jüngere, alleinstehende und weniger gebildete Menschen stärker stigmatisiert [108]. Umgekehrt wurde ein signifikanter Zusammenhang zwischen einem geringeren Grad an Stigmatisierung und grösserem Wissen über Pflege und Versorgung festgestellt [109]. Dies bedeutet in der Praxis, dass es Bemühungen bedarf, die Stigmatisierung von Haushalten mit HIV-Infizierten abzubauen, um so das Wissen und die Methoden im Bereich der Versorgung und Pflege von Kindern zu verbessern und bessere Outcomes bei Kindern zu erzielen.

Zusammengenommen belasten die Faktoren der Isolation aufgrund von niedrigem Einkommen, geographischer Lage und HIV/AIDS-bedingter Stigmatisierung die kindliche Gesundheit. Die oben aufgeführten Strategien zur Unterstützung von Pflege und Versorgung beruhen ebenfalls auf lokaler Planung und der lokalen Entwicklung von Ressourcen. Soweit die betroffenen Gemeinden jedoch die Möglichkeit haben, selbst in Erscheinung zu treten, sollten sie ergänzend fordern, dass ihre Rechte auf Gesundheit von den städtischen, staatlichen, nationalen und internationalen Behörden erfüllt werden. In den Slums (Favelas) von Rio de Janeiro, Brasilien, wird dies durch die nicht-staatliche Organisation CEDAPS (Zentrum für die Gesundheitsförderung) beispielhaft vorgelebt [110]. Diese Bewegung auf Gemeindeebene hat es geschafft, dass sich die Menschen dort mit den sozialen Massnahmen identifizieren, welche die Determinanten der lokalen Vulnerabilität ins Visier nehmen, und schärft gleichzeitig das Bewusstsein der Menschen für ihr Recht auf Gesundheit.

\section{Schlussfolgerungen und Ausblicke}

Würde die Armut als Hintergrund und grundlegendes Merkmal der HIV/AIDS-Epidemie nicht so überhandnehmen, wären die Probleme im Bereich der Ernährung und Gesundheit nicht ganz so ernst. In einer Welt, in der die ganz grosse Armut immer noch nicht besiegt ist, gilt es anscheinend, eine hohe Hürde zu überwinden, da für eine «nachhaltige Entwicklung diverse politische, wirtschaftliche und soziale Änderungen notwendig sind, u.a. die Ermächtigung der Entrechteten, die Erweiterung des Kreises, der Zugang auf Vermögenswerte und andere Ressourcen hat, die Annäherung von Arm und Reich und die Anpassung an ein umweltgerechtes Konsumverhalten» [111].

Im Rahmen des anspruchsvollen Strebens nach einer nachhaltigen Entwicklung stellen HIV/AIDS eine immense und wachsende Herausforderung für den Ideenreichtum des öffentlichen Gesundheitswesens, eine grosse Belastung für die Ressourcen und eine der Ursachen für unermessliches Leid und Stigmatisierung dar. Das AIDS-Zeitalter dauert nun schon ein Vierteljahrhundert an. Die hier vorliegende Analyse hat den gesellschaftlichen Ursprung der Probleme im Bereich der Gesundheitsversorgung und Ernährung aufgezeigt. Aufgrund der langen Latenzzeit bis zum Ausbruch der Erkrankung und deren Progression bis zum Tod, war es für uns schwierig, die diversen gesundheitlichen und sozialen Anforderungen zu verstehen und entsprechend darauf zu reagieren.

Die HIV/AIDS-Pandemie ist erst ein Vierteljahrhundert alt und ihre epidemiologischen und pharmakologischen Aspekte entwickeln und verändern sich weiter. Wir haben für diese Analyse eine Unmenge an Literatur gesichtet, um die zentrale Rolle aufzuzeigen, die Ernährung, Nahrung und Ernährungssicherheit bei den Bemühungen spielen, die Auswirkungen der Infektion abzuschwächen. Die Erfahrungen und Argumente haben uns jedoch die Grenzen der Entwicklung evidenzba- 
sierter Interventionen aufgezeigt, die der einzig mögliche Ansatz sind, um sich den Unzulänglichkeiten in der Kinderpflege und -versorgung und den klaffenden Lücken der Ernährungsunsicherheit zuzuwenden. Unsere Wissenslücken wurden identifiziert und zeigen die Hauptschwerpunkte weiterer Forschungsarbeiten, um die Auswirkungen von HIV/AIDS weiter abzuschwächen. Tabelle 6 enthält einige von einem Expertengremium der WHO-Beratung festgelegte Fragen aus der praktischen Forschung [74]. Am stärksten betroffen von HIV/AIDS sind jedoch die ressourcenschwächsten Regionen, in denen entsprechende Präventions-, Linderungs- und Pflegebemühungen noch immer einen Luxus darstellen. Vor dem Hintergrund begrenzter Finanzmittel konkurrieren die für die Forschung aufgewendeten Gelder direkt mit den für Interventionen benötigten Ressourcen.

Trotz der Bemühungen, die Wissenslücken und Schwerpunkte weiterer Forschungsarbeiten zu identifizieren, folgerte das WHO-Gremium, dass viel zur Verbesserung der Gesundheit, des Ernährungsstatus und der Lebensqualität der mit HIV/AIDS lebenden Menschen getan werden kann und sollte [74]. Die meisten Mitarbeiter auf Programmebene $[43,96]$ und die meisten Akademiker, die Politiker beraten und informieren $[62,89,92]$, raten dazu, Erfahrungen zu sammeln und die Methoden im Rahmen der Formulierung kurz- und langfristiger Massnahmen zum Thema Ernährungssicherheit zu verfeinern. Zwei der wichtigsten Grundsätze, die sich wie ein roter Faden durch diesen Übersichtsartikel ziehen, sind: (1) Es bedarf eines umfassenden und ganzheitlichen Ansatzes zur Behandlung der grundsätzlichen Probleme, die über die HIVInfizierten hinausgehen, und (2) es bedarf der Basisarbeit auf Gemeindeebene, welche die Beratung, die aktive Beteiligung und die nachhaltige Verantwortung von Gemeinden umfasst.

Was den Zusammenhang zwischen der Pflege und Versorgung der Kinder und ihrer Gesundheit betrifft, so sind die durch die HIV/AIDS-Epidemie bedingten Probleme vielfach die gleichen, die auch die Ernährungssicherheit beeinflussen. Das Gleichgewicht der Haushalte wird gestört, Einkommen und gesparte Ressourcen gehen verloren und die Versorgung der Kinder obliegt zunehmend Personen, die entweder zu jung und unerfahren oder zu alt und schwach sind, um diese Aufgabe richtig zu erfüllen. Die traditionellen Methoden der Kinderpflege und -versorgung der Gesellschaften sind jedoch nicht immer auf das Überleben aller Kinder ausgerichtet. Pathogene, die durch unzureichende Hygiene in Nahrung und Wasser gelangen, können für mangelernährte Kinder mit einem beeinträchtigten Immunsystem lebensbedrohlich sein. Moss et al. [112] empfehlen die Ausarbeitung «evidenzbasierter, an die lokalen Gegebenheiten angepasster Richtlinien zur Kinderpflege und -versorgung»; in diesem Fall wiederum bleibt die Antwort auf die Frage hängig, wie die Ressourcen am besten zwischen der tatsächlichen Erbringung von Leistungen und den diesbezüglichen Forschungsarbeiten aufzuteilen sind.

Da uns keine Finanzmittel für eine engagierte investigative Forschung zur Verfügung stehen, um die noch bestehenden Lücken in unserem Grundwissen und in unserem praktischen Wissen zu schliessen, können wir zumindest die Lücken in unserem praktischen Wissen über die vor Ort gesammelten praktischen Erfahrungen behandeln. Auf Konferenzen, wie
Tabelle 6. Sachdienliche Forschungsfragen, um die bestehenden Wissenslücken bezüglich der Zusammenhänge zwischen Ernährungssicherheit und HIV/AIDS zu schliessen

1 Wie wirken sich die verbesserte Lebensmitteltechnologie auf Haushaltsebene, Ernährungsberatung und Bereitstellung von Nahrungsmitteln, die im Rahmen von Pflege- und Versorgungsmassnahmen auf Gemeindeebene verteilt werden, auf die Verbesserung des Ernährungsstatus, die Progression der Erkrankung und die Lebensqualität aus?

2 Inwieweit sollten die Programme der Nahrungsmittel- und Ernährungshilfe angesichts der HIV/AIDS-Pandemie verändert werden? Sollten beispielsweise die Grösse oder Zusammensetzung der Rationen für HIV-Infizierte geändert werden? Welche Kriterien müssen Lebensmittel erfüllen, um die Auswirkungen von HIV/AIDS zu lindern?

3 Welche Ernährungsinterventionen können die Ernährungsauswirkungen von HIV, die sich aufgrund der Abnahme der landwirtschaftlichen Produktivität bzw. der Versorgungskapazität ergeben, im Hinblick auf die Ernährungssicherheit erfolgreich lindern?

4 Wie wirkt sich die antiretrovirale Therapie auf mangelernährte Populationen aus? Beeinflusst der Ernährungsstatus die Wirksamkeit der Therapie und das Risiko bzw. den Schweregrad der mit der Therapie verbundenen unerwünschten Nebenwirkungen? Würden Ernährungsinterventionen, die begleitend zur antiretroviralen Therapie verabreicht werden - insbesondere in unterernährten Populationen und bei stillenden Müttern - die gesundheitlichen Outcomes verbessern?

Auszug aus der WHO [70].

z.B. dem Afrika-Forum [96], hat sich gezeigt, dass sich eine allgemeine Vorstellung darüber, welche Massnahmen funktionieren, gut funktionieren oder nicht funktionieren, über die kollektiven Erfahrungen der in der Praxis tätigen Mitarbeiter herausfiltern lässt. Die Anpassung der Interventionen an die regionalen Gegebenheiten und an die sich ändernden Realitäten in der Praxis gilt als positive Tugend. Derzeit konzentriert man sich eher darauf, ganzheitliche Programme für HIV/ AIDS, Nahrungsmittel- und Ernährungssicherheit zu erarbeiten, die sich positiv auf die Lebensmittelsicherheit und die Gesundheit der Kinder auswirken.

Die Wirklichkeit vor Ort, in den Gemeinden, wo Gemeindearbeiter, die unter Personalmangel, unzureichender Ausstattung und ungenügenden Finanzmitteln leiden, und Bewohner täglich um Nahrung und Pflege kämpfen müssen, ist in der Tat dadurch gekennzeichnet, dass die Bewältigungsmechanismen extremen Belastungen ausgesetzt sind und kurz vor dem Zusammenbruch stehen.

Es gilt jedoch Visionen zu entwickeln, die über den Status quo hinausgehen. Wenn wir uns darauf einigen können, dass der Begriff Ernährungssicherheit im Allgemeinen quantitativ ist und dafür steht, dass ein Mensch über genügend Nahrung verfügt, um Hunger und Unterernährung zu vermeiden, müssen sowohl kurz-als auch langfristig für die Menschen, die mit HIV/AIDS leben, andere Gesichtspunkte einer qualitativ hochwertigen Ernährung zutage treten [113]. Die Lebensmittelsicherheit, d.h. Nahrung, die frei von Verunreinigungen ist, ist unmittelbar von Bedeutung, da die durch Lebensmittel hervorgerufenen Krankheiten sich bei Menschen mit einer geschwächten Immunabwehr möglicherweise sehr viel schlim- 
mer auswirken. Wie bereits an anderer Stelle bezüglich der Überernährung besprochen, geht mit Einführung der Therapie für manche der serumpositiven Populationen das Schreckgespenst um, dass das langfristige Überleben mit vielen Problemen, auch die Gesundheit der Patienten langfristig zu gewährleisten, verbunden sein wird. Als ultimative Verbesserung gilt es, über eine ausreichende Menge verträglicher Lebensmittel hinaus, eine gesunde Ernährung in Betracht zu ziehen, welche die Risiken für nicht ansteckende Krankheiten senkt. Dies ist ganz im Sinne von Gopalan [47], der sehr weise anmerkt, dass Ernährungssicherheit auf Haushaltsebene mehr sei als die blosse Vermeidung chronischen Hungers und dass es das Ziel der Politiker in Entwicklungsländern sein sollte, im nächsten Jahrhundert nicht nur das reine Überleben, sondern eine angemessene Ernährung zu gewährleisten. Dies wäre für alle Gemeinden, in denen Menschen mit HIV/AIDS leben, eine optimistischere Zukunftsperspektive.

\section{Literatur}

1 Gottlieb MS, Schanker HM, Fan PT, Weisman JT: Pneumocystis pneumonia - Los Angeles. MMWR 1981;30:1-3.

-2 Heeney JL, Dalgleish AG, Weiss RA: Origins of HIV and the evolution of resistance to AIDS. Science 2006;313:462-466.

3 Piot P, Bartos M, Ghys PD, et al: The global impact of HIV/AIDS. Nature 2001;410:968973.

4 Shears P: Cholera. Ann Trop Med Parasitol 1994;88:109-122.

5 Guerrant RL, Carneiro-Filho BA, Dillingham RA: Cholera, diarrhea, and oral rehydration therapy: triumph and indictment. Clin Infect Dis 2003;37:398-405.

6 Henderson DA: Smallpox eradication. Public Health Rep 1980;95:422-426.

7 Zlotnik H: Demographic trends; in Caballero B, Popkin BM (eds): The Nutrition Transition: Diet and Disease in the Developing World. London, Academic Press, 2002, pp 71-107.

8 Rutengwe RM: Identifying strategic interventions for improving household food and nutrition security in an urban informal settlement, South Africa. Asia Pac J Clin Nutr 2004; 13(suppl):S169.

9 Walker N., Schwartländer J: Meeting international goals in child survival and HIV/AIDS. Lancet 2002;360:284-289.

10 Hunter S, Williamson J: Children on the Brink 2000: Executive Summary - Updated Estimates and Recommendations for Intervention. The Synergy Project 2000. Washington, USAID, 2000, pp 1-32.

11 Somerville MA, Orkin AJ: Human rights, discrimination and AIDS: concepts and issues. AIDS 1989;3(suppl 1):S283-S287.

12 Hecht R, Alban A, Taylor K, et al: Putting it together: AIDS and the millennium development goals. PLoS Med 2006;3:e455.

13 Haddad L: No longer off the menu: the welcome re-emergence of food on the nutrition agenda. Forum Nutr 2003;56:383-384.

14 The President's Emergency Plan for AIDS Relief. Report on Food and Nutrition for People Living with HIV/AIDS. Washington, Office of the US Global AIDS Coordinator, US Department of State, 2006.

15 Scrimshaw NS, Taylor CE, Gordon JE: Interactions of nutrition and infection. Am J Med Sci 1959;237:367-403.
16 Scrimshaw NS, Taylor CE, Gordon JE: Interactions of nutrition and infection. Monogr Ser World Health Organ 1968;57:3-329.

17 Keusch GT, Farthing MJG: Nutrition and infection. Annu Rev Nutr 1986;6:131-154.

18 Scrimshaw NS, SanGiovanni JP: Synergism of nutrition, infection, and immunity: an overview. Am J Clin Nutr 1997;66:464S-477S

19 Wanke CA, Silva M, Knox TA, et al: Weight loss and wasting remain common complications in individuals infected with human immunodeficiency virus in the era of highly active antiretroviral therapy. Clin Infect Dis 2000;31:803-805

20 Ungar BLP, Ward DJ, Fayer R, Quinn CA: Cessation of cryptosporidium-associated diarrhea in an acquired immunodeficiency syndrome patient after treatment with hyperimmune bovine colostrum. Gastroenterology 1990:98:486-489.

-21 Saini A, Nasser AS, Stewart CE: Waste management - cytokines, growth factors and cachexia. Cytokine Growth Factor Rev 2006;7: 475-486.

22 MacDonald KS, Malonza I, Chen DK, et al: Vitamin A and risk of HIV-1 seroconversion among Kenyan men with genital ulcers. AIDS 2001; 15:635-639.

23 Semba RD, Neville MC: Breast-feeding, mastitis, and HIV transmission: nutritional implications. Nutr Rev 1999;57:146-153.

24 Fawzi WW: Nutritional factors and vertical transmission of HIV-1: epidemiology and potential mechanisms. Ann NY Acad Sci 2000; 918:99-114.

25 Dreyfuss ML, Fawzi WW: Micronutrients and vertical transmission of HIV-1. Am J Clin Nutr 2002;75:959-970.

26 Downs JH, Cooper PA: HIV and lactation. Ann Nestlé [Engl] 2007;65:29-38.

27 Tang AM, Graham NM, Kirby AJ, et al: Dietary micronutrient intake and risk progression to acquired immunodeficiency syndrome (AIDS) in human immunodeficiency virus type (HIV-1)-infected homosexual men. Am J Epidemiol 1993;138:937-951.

28 Tang AM, Graham NM, Saah AJ: Effects of micronutrient intake on survival in human immunodeficiency virus type I infection. Am J Epidemiol 1996;143:1244-1256.
29 Tang A, Graham N, Semba R, Saah A: Association between serum vitamin $A$ and $E$ levels and HIV-1 disease progression. AIDS 1997;11: 613-620.

30 Baum MK, Shor-Posner G, Lu Y, et al: Micronutrients and HIV-1 disease progression. AIDS 1995;9:1051-1056.

31 Mehta S, Finkelstein JL, Fawzi W: Nutritional interventions in HIV-infected breastfeeding women. Ann Nestlé [Engl] 2007;65:39-48.

32 Beck MA, Kolbeck PC, Shi Q, et al: Increased virulence of a human enterovirus (coxsackievirus B3) in selenium-deficient mice. J Infect Dis 1994;170:351-357.

33 Beck MA, Handy J, Levander OA: Host nutritional status: the neglected virulence factor. Trends Microbiol 2004;12:417-423.

34 Nelson HK, Shi Q, Van Dael P, et al: Host nutritional selenium status as a driving force for influenza virus mutations. FASEB J 2001;15: 1846-1848.

35 Beck MA, Nelson HK, Shi Q, et al: Selenium deficiency increases the pathology of in influenza virus infection. FASEB J 2001;15:14811486.

36 Sazawal S, Black RE, Ramsan M, et al: Effects of routine prophylactic supplementation with iron and folic acid on admission to hospital and mortality in preschool children in a high malaria transmission setting: communitybased, randomised, placebo-controlled trial. Lancet 2006;367:133-143.

37 Weinberg ED: Iron withholding: a defense against viral infections. Biometals 1996;9: 393-399.

38 Bobat R, Coovadia H, Stephen C, et al: Safety and efficacy of zinc supplementation for children with HIV-1 infection in South Africa: a randomised double-blind placebo-controlled trial. Lancet 2006:367:814-815.

39 Olsen A, Mwaniki D, Krarup H, Friis H: Lowdose iron supplementation does not increase HIV-1 load. J Acquir Immune Defic Syndr 2004;36:637-638.

40 Friis H (ed): Micronutrients and HIV Infection. Boca Raton, CRC Press, 2002.

41 Piot P: Keynote Address. Nutrition and HIV/ AIDS. Nutrition Policy Paper No. 20. SubCommittee on Nutrition. Geneva, SCN 2001, pp 7-10. 
-42 Whiteside A, Barnett T, George G, Van Niekerk AA: Through a glass, darkly: data and uncertainty in the AIDS debate. Developing World Bioeth 2003;3:49-76.

-43 Tabi M, Vogel RL: Nutritional counselling: an intervention for HIV-positive patients. J Adv Nurs 2006;54:676-682.

-44 Fields-Gardner C, Fergusson P; American Dietetic Association; Dietitians of Canada: Position of the American Dietetic Association and Dietitians of Canada: nutrition intervention in the care of persons with human immunodeficiency virus infection. J Am Diet Assoc 2004;104:1425-1441.

-45 Tontisirin K, Bhattacharjee L: Food security and nutrition - a household perspective. Forum Nutr 2003;56:382-383.

46 Casey PH, Simpson PM, Gossett JM, et al: The association of child and household food insecurity with childhood overweight status. Pediatrics 2006;118:e1406-e1413.

47 Gopalan C: Achieving household nutrition security in societies in transition: an overview. Asia Pac J Clin Nutr 2001;10(suppl):S4S12.

-48 Kendall A, Olson CM, Frongillo EA Jr: Validation of the Radimer/Cornell measures of hunger and food insecurity. J Nutr 1995; 125:27932801.

-49 Frongillo EA Jr, Rauschenbach BS, Olson CM, et al: Questionnaire-based measures are valid for the identification of rural households with hunger and food insecurity. J Nutr 1997;127: 699-705.

-50 Onyango AW: Dietary diversity, child nutrition and health in contemporary African communities. Comp Biochem Physiol A Mol Integr Physiol 2003;136:61-69.

51 Keenan DP, Olson C, Hersey JC, Parmer SM: Measures of food insecurity/security. J Nutr Educ 2001;33(suppl 1):S49-S58.

52 Rose D: Economic determinants and dietary consequences of food insecurity in the United States. J Nutr 1999;129(suppl):517S-520S.

-53 Shariff ZM, Khor GL: Obesity and household food insecurity: evidence from a sample of rural households in Malaysia. Eur J Clin Nutr 2005;59:1049-1058.

54 Webb P, Coates J, Frongillo EA, et al: Measuring household food insecurity: why it's so important and yet so difficult to do. J Nutr 2006 136:1404S-1408S

55 Nyindo M: Complementary factors contributing to the rapid spread of HIV-I in sub-Saharan Africa: a review. East Afr Med J 2005;82: $40-46$.

56 Mhalu FS: Burden of diseases in poor resource countries: meeting the challenges of combating HIV/AIDS, tuberculosis and malaria. Tanzan Health Res Bull 2005;7:179-184.

57 Chopra M, Darnton-Hill I: Responding to the crisis in sub-Saharan Africa: the role of nutrition. Public Health Nutr 2006;9:544-550.

58 Watts J: Targets now set by G8 countries to reduce 'diseases of poverty'. Lancet 2000;356: 408.

59 Rukuni M: Africa: addressing growing threats to food security. J Nutr;132:3443S-3448S.

HIV, Ernährungssicherheit und

Gesundheit von Kindern

Gesundheit von Kindern
60 Saasa OS: HIV/AIDS and Development - Unsolved Challenges for Africa. Nutrition and HIV/AIDS. Nutrition Policy Paper No. 20. SubCommittee on Nutrition. Geneva, SCN, 2001, pp 19-27.

61 Hunter SS, Bulirwa E, Kisseka E: AIDS and agricultural production. Report of a land utilization survey, Masaka and Rakai districts of Uganda. Land Use Policy 1993;10:241-258.

62 Gillespie S, Haddad L, Jackson R: HIV/AIDS, food and nutrition security: impacts and actions. 28th Session of the ACC/SCN Symposium on Nutrition and HIV/AIDS, Nairobi, 2001.

63 Cummings B, Mengistu M, Negash W, et al: Barriers to and facilitators for female participation in an HIV prevention project in rural Ethiopia: findings from a qualitative evaluation. Cult Health Sex 2006;8:251-266.

64 Hindin MJ: Women's input into household decisions and their nutritional status in three resource-constrained settings. Public Health Nutr 2006;9:485-493.

65 Kruger A, Lemke S, Phometsi M, et al: Poverty and household food security of black South African farm workers: the legacy of social inequalities. Public Health Nutr 2006;9: 830-836.

66 Thomas F: Stigma, fatigue and social breakdown: exploring the impacts of HIV/AIDS on patient and carer well-being in the Caprivi Region, Namibia. Soc Sci Med 2006;63:31743187.

67 Thomas BE, Rehman F, Suryanarayanan D, et al: How stigmatizing is stigma in the life of people living with HIV: a study on HIV positive individuals from Chennai, South India. AIDS Care 2005;17:795-801.

68 Mawar N, Saha S, Pandit A, Mahajan U: The third phase of HIV pandemic: social consequences of HIV/AIDS stigma and discrimination and future needs. Indian J Med Res 2005; 122:471-484.

69 Reidpath DD, Brijnath B, Chan KY: An Asia Pacific six-country study on HIV-related discrimination: introduction. AIDS Care 2005; 17(suppl 2):S117-S127.

70 VanLandingham MJ, Im-Em W, Saengtienchai C: Community reaction to persons with HIV/AIDS and their parents: an analysis of recent evidence from Thailand. J Health Soc Behav 2005;46:392-410.

71 Gillespie S, Haddad L, Jackson R: HIV/AIDS, Food and Nutrition Security: Impacts and Actions. Nutrition and HIV/AIDS. Nutrition Policy Paper No. 20. SubCommittee on Nutrition. Geneva, SCN, 2001, pp 31-45.

72 Wyss K, Hutton G, N’Diekhor Y: Costs attributable to AIDS at household level in Chad. AIDS Care 2004;16:808-816.

73 Food and Nutrition Board, Institute of Medicine: Dietary Reference Intakes for Energy, Carbohydrate, Fiber, Fat, Fatty Acids, Cholesterol, Protein, and Amino Acids. Part 1, Summary and chapters 1-9. Washington, National Academy Press, 2002.

74 World Health Organization: Nutrient Requirements for People Living with HIB/AIDS. Report of Technical Consultation. Geneva, WHO, 2003.
5 Manuela de Paoli M, Manongi R, Klepp KI: Are infant feeding options that are recommended for mothers with HIV acceptable, feasible, affordable, sustainable and safe? Pregnant women's perspectives. Public Health Nutr 2004;7:611-619.

76 Thior I, Lockman S, Smeaton LM, et al: Breastfeeding plus infant zidovudine prophylaxis for 6 months vs formula feeding plus infant zidovudine for 1 month to reduce mother-tochild HIV transmission in Botswana: a randomized trial: the Mashi Study. JAMA 2006; 296:794-805

77 Forrester JE, Tucker KL, Gorbach SL: Dietary intake and body mass index in HIV-positive and HIV-negative drug abusers of Hispanic ethnicity. Public Health Nutr 2004;7:863870.

78 Paton NI, Sangeetha S, Earnest A, Bellamy R: The impact of malnutrition on survival and the CD4 cell response in HIV-infected patients starting antiretroviral therapy. HIV Med 2006; 7:323-330.

79 Kim JH, Spiegelman D, Rimm E, Gorbach SL The correlates of dietary intake along HIVpositive adults. Am J Clin Nutr 2001;74:852861.

80 Betsi NA, Koudou BG, Cisse G, et al: Effect of an armed conflict on human resources and health systems in Cote d'Ivoire: prevention of and care for people with HIV/AIDS. AIDS Care 2006; 18:356-365.

81 Spiegel PB: HIV/AIDS among conflict-affected and displaced populations: dispelling myths and taking action. Disasters 2004;28: 322-339.

82 Vorster HH, Venter CS, Wissing MP, Margetts BM: The nutrition and health transition in the North West Province of South Africa: a review of the THUSA (Transition and Health during Urbanisation of South Africans) study. Public Health Nutr 2005;8:480-490.

83 Bourne LT, Lambert EV, Steyn K: Where does the black population of South Africa stand on the nutrition transition? Public Health Nutr 2002;5:157-162.

84 Zimmet P: Epidemiology of diabetes mellitus and associated cardiovascular risk factors: focus on human immunodeficiency virus and psychiatric disorders. Am J Med 2005;118(sup$\mathrm{pl} 2): 3 \mathrm{~S}-8 \mathrm{~S}$

85 Amorosa V, Synnestvedt M, Gross R, et al: A tale of 2 epidemics: the intersection between obesity and HIV infection in Philadelphia. J Acquir Immune Defic Syndr 2005;39:557561.

86 Jaime PC, Florindo AA, Latorre MD, Segurado AA: Central obesity and dietary intake in HIV/AIDS patients. Rev Saude Publica 2006; 40:634-640.

87 Rosegrant MW, Cline SA: Global food security: challenges and policies. Science 2003; 302:1917-1919.

88 Gregory PJ, Ingram JS, Brklacich M: Climate change and food security. Philos Trans R Soc Lond B Biol Sci 2005;360:2139-2148. 
89 Gillespie S: AIDS, Poverty and Hunger: Challenges and Response. Food Policy Statement. Washington, International Food Policy Research Institute, 2006.

90 Gillespie S: Interventions and impacts: how HIV/AIDS interacts with food and nutrition security: an overview to volume 1 . Int Conf HIV/AIDS and Food and Nutrition Security, Durban, 2005.

91 Gillespie S: Policy and program response: responding to the Interactions between HIV/ AIDS an food and nutrition security: an overview to volume 2. Int Conf HIV/AIDS and Food and Nutrition Security, Durban, 2005.

92 Parley PG, Alston M, Piggett RR: Agricultural R\&D in the Developing World, Too Little Too Late? Washington, International Food Policy Research Institute, 2006.

93 Kadiyala S, Gillespie S: Rethinking food aid to fight AIDS. Food Nutr Bull 2004;25:271-282.

94 World Food Programme: Food Security, Food Aid and HIV/AIDS: Project Ideas to Address the HIV/AIDS Crisis. Rome, WFP, 2001.

95 Marchione TJ: Interactions with the recipient community in targeted food and nutrition programs. J Nutr 2005; 135:886-889.

96 The African Forum 2006: An Integrated Response to the Dual Epidemic of HIV/ AIDS and Food Insecurity, Lukasa, Zambia, 2006.

97 Martin-Prevel Y: 'Care' and public nutrition. Sante 2002;12:86-93.
98 Engle PL, Bentley M, Pelto G: The role of care in nutrition programmes: current research and a research agenda. Proc Nutr Soc 2000;59:25-35.

99 Ghosh S, Shah D: Nutritional problems in urban slum children. Indian Pediatr 2004; 41:682-696.

100 Shetty AK, Powell G: Children orphaned by AIDS: a global perspective. Semin Pediatr Infect Dis 2003; 14:25-31.

101 Oleke C, Blystad A, Rekdal OB: 'When the obvious brother is not there': political and cultural contexts of the orphan challenge in northern Uganda. Soc Sci Med 2005;61: 2628-2638.

102 Nyambedha EO, Wandibba S, AagaardHansen J: Policy implications of the inadequate support systems for orphans in western Kenya. Health Policy 2001;58:83-96.

103 Engle PL, Pelto G, Bentley P: Care for nutrition and development. J Indian Med Assoc 2000;98:530-535.

104 White J, Robinson E: HIV/AIDS and Rural Livelihoods in Sub-Saharan Africa. Policy Series 6. Chatham, Natural Resources Institute, 2000.

105 Lapping K, Marsh DR, Rosenbaum J, et al: The positive deviance approach: challenges and opportunities for the future. Food Nutr Bull 2002;23(suppl):130-137.

106 Marsh DR, Sternin M, Khadduri R, et al: Identification of model newborn care practices through a positive deviance inquiry to guide behavior-change interventions in Haripur, Pakistan. Food Nutr Bull 2002; 23(suppl):109-118.
107 Ha PB, Bentley ME, Pachon H, et al: Caregiver styles of feeding and child acceptance of food in rural Viet Nam. Food Nutr Bull 2002;23(suppl):95-100.

108 Hamra M, Ross MW, Orrs M, D’Agostino A: Relationship between expressed HIV/AIDSrelated stigma and HIV-beliefs/knowledge and behaviour in families of HIV infected children in Kenya. Trop Med Int Health 2006;11:513-527.

109 Hamra M, Ross MW, Karuri K, et al: The relationship between expressed HIV/AIDS-related stigma and beliefs and knowledge about care and support of people living with AIDS in families caring for HIV-infected children in Kenya. AIDS Care 2005;17:911922.

110 Edmundo K, Guimaraes W, Vasconcelos Mdo S, et al: Network of communities in the fight against AIDS: local actions to address health inequities and promote health in Rio de Janeiro, Brazil. Promot Educ 2005;suppl 3:15-19.

111 Struble MB, Aomari LL: Position of the American Dietetic Association: addressing world hunger, malnutrition, and food insecurity. J Am Diet Assoc 2003;103:10461057.

112 Moss WJ, Ramakrishnan M, Storms D, et al: Child health in complex emergencies. Bull World Health Organ 2006;84:58-64.

113 Lobstein T: Food policies: a threat to health? Proc Nutr Soc 2002;61:579-585. 